\title{
The ALS-linked E102Q mutation in Sigma receptor-1 leads to ER stress-mediated defects in protein homeostasis and dysregulation of RNA-binding proteins
}

\author{
Alice Dreser ${ }^{1}$, Jan Tilmann Vollrath ${ }^{1}$, Antonio Sechi ${ }^{2}$, Sonja Johann ${ }^{3}$, Andreas Roos ${ }^{1,4,5}$, Alfred Yamoah ${ }^{1}$, Istvan Katona ${ }^{1}$, \\ Saeed Bohlega ${ }^{6}$, Dominik Wiemuth ${ }^{7}$, Yuemin Tian ${ }^{7}$, Axel Schmidtt ${ }^{7}$, Jörg Vervoorts ${ }^{8}$, Marc Dohmen ${ }^{8}$, Cordian Beyer ${ }^{3}$, Jasper Anink ${ }^{9}$, \\ Eleonora Aronica ${ }^{9}$, Dirk Troost ${ }^{9}$, Joachim Weis ${ }^{1,10}$ and Anand Goswami ${ }^{*, 1,10}$
}

Amyotrophic lateral sclerosis (ALS) is characterized by the selective degeneration of motor neurons (MNs) and their target muscles. Misfolded proteins which often form intracellular aggregates are a pathological hallmark of ALS. Disruption of the functional interplay between protein degradation (ubiquitin proteasome system and autophagy) and RNA-binding protein homeostasis has recently been suggested as an integrated model that merges several ALS-associated proteins into a common pathophysiological pathway. The E102Q mutation in one such candidate gene, the endoplasmic reticulum (ER) chaperone Sigma receptor-1 (SigR1), has been reported to cause juvenile ALS. Although loss of SigR1 protein contributes to neurodegeneration in several ways, the molecular mechanisms underlying E102Q-SigR1-mediated neurodegeneration are still unclear. In the present study, we showed that the E102Q-SigR1 protein rapidly aggregates and accumulates in the ER and associated compartments in transfected cells, leading to structural alterations of the ER, nuclear envelope and mitochondria and to subsequent defects in proteasomal degradation and calcium homeostasis. ER defects and proteotoxic stress generated by E102Q-SigR1 aggregates further induce autophagy impairment, accumulation of stress granules and cytoplasmic aggregation of the ALS-linked RNAbinding proteins (RBPs) matrin-3, FUS, and TDP-43. Similar ultrastructural abnormalities as well as altered protein degradation and misregulated RBP homeostasis were observed in primary lymphoblastoid cells (PLCs) derived from E102Q-SigR1 fALS patients. Consistent with these findings, lumbar $\alpha$-MNs of both sALS as well as fALS patients showed cytoplasmic matrin-3 aggregates which were not co-localized with pTDP-43 aggregates. Taken together, our results support the notion that E102Q-SigR1-mediated ALS pathogenesis comprises a synergistic mechanism of both toxic gain and loss of function involving a vicious circle of altered ER function, impaired protein homeostasis and defective RBPs.

Cell Death and Differentiation (2017) 24, 1655-1671; doi:10.1038/cdd.2017.88; published online 16 June 2017

Several familial ALS (fALS) and frontotemporal dementia (FTD)-linked mutations affect protein homeostasis and autophagy, including ubiquilin-2 (UBQLN2), p62/SQSTM1 (sequestosome1), optineurin (OPTM), TANK-binding kinase 1 (TBK1), valosin-containing protein ( $V C P$ ), charged multivesicular body protein 2B (CHMP2B), vesicle-associated membrane protein B (VAPB) and FIG4., ${ }^{1,2}$ Recent studies emphasized a functional interplay between autophagy and RNA processing, associating several ALS-FTD genes in a converging pathway leading to insufficient degradation and abnormal aggregation of proteins. ${ }^{2}$ Specifically, mutations in RBPs such as Tar DNAbinding protein-43 (TDP-43), fused in sarcoma (FUS), survival of motor neuron-1 (SMN1), ataxin-2 (ATX2), optineurin (OPT), angiogenin (ANG) and matrin-3 (MATR3) cause fALS with distinct aggregates, ${ }^{3}$ disturbed autophagy and altered RNA processing. Even in sporadic ALS (sALS), toxic aggregates of such proteins in MNs are frequent and can be triggered by defective UPS/autophagy. ${ }^{4}$

Aggregation of these RBPs together with other proteins is physiological and reversible (unfolded protein response, UPR). ${ }^{5}$ RBPs control RNA polymerase elongation, mRNA maturation, transport and degradation, and regulate transcriptional activity and distribution of RNAs by RNA granule formation. Among the latter, stress granules (SGs) are generated in response to stressful conditions. Prolonged SG formation affects protein quality control and vital cellular processes like apoptosis, signalling and RNA decay. ${ }^{5}$

Sigma receptor-1 (SigR1) is an ER chaperone involved in neuronal survival, ion channel activity, $\mathrm{Ca}^{2+}$ signalling, synaptic plasticity, memory and drug addiction. ${ }^{6}$ The E102Q-SigR1

\footnotetext{
${ }^{1}$ Institute of Neuropathology, RWTH Aachen University Medical School, Aachen, Germany; ${ }^{2}$ Institute of Biomedical Engineering, Deparment of Cell Biology, RWTH Aachen University Medical School, Aachen, Germany; ${ }^{3}$ Institute of Neuroanatomy, RWTH Aachen University Medical School, Aachen, Germany; ${ }^{4}$ Leibniz-Institut für Analytische Wissenschaften - ISAS - e.V., Dortmund, Germany; ${ }^{5}$ Institute of Genetic Medicine, John Walton Muscular Dystrophy Research Centre, International Centre for Life, Central Parkway, Newcastle upon Tyne, England, UK; ${ }^{6}$ Department of Genetics, King Faisal Specialist Hospital and Research Centre, Riyadh, Saudi Arabia; ${ }^{7}$ Institute of Physiology, RWTH Aachen University Medical School, Aachen Germany; ${ }^{8}$ Institute of Biochemistry and Molecular Biology, RWTH Aachen University Medical School, Aachen, Germany and ${ }^{9}$ Department of (Neuro)Pathology, Academic Medical Center, University of Amsterdam, Amsterdam, The Netherlands

*Corresponding author: A Goswami, Institute of Neuropathology, RWTH Aachen University Medical School, Pauwelsstrasse 30, Aachen 52074, Germany. Tel/Fax: +49 241 8037361; E-mail: agoswami@ukaachen.de

${ }^{10}$ These authors contributed equally to this paper.

Received 11.11.15; revised 14.3.17; accepted 22.3.17; Edited by D Rubinsztein; published online 16.6.17
} 
missense mutation causes $\mathrm{fALS} ;{ }^{7}$ a splice site mutation (c.151 $+1 G>T)^{8}$ and recently the homozygous $E 138 Q$ and $E 150 K$ mutations ${ }^{9}$ have been linked to autosomal recessive distal hereditary motor neuropathy (dHMN). Moreover, SigR1 is involved in several neurodegenerative disorders including Parkinson's, Alzheimer's and Huntington's disease. ${ }^{10}$
We previously described abnormal modification as well as altered localization of SigR1 protein in SALS patient spinal cord. ${ }^{11}$ Mavlyutov et al. demonstrated that lack of SigR1 exacerbates ALS progression in G93A-SOD1 mice. ${ }^{12}$ SigR $1^{-/-}$mice showed MND pathology and symptoms. ${ }^{13}$ In vitro, SigR1 depletion causes $\mathrm{Ca}^{2+}$ dysregulation, a

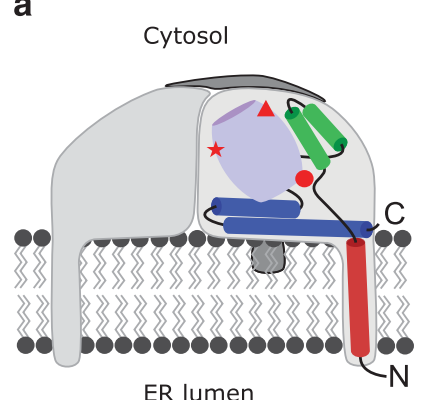

b

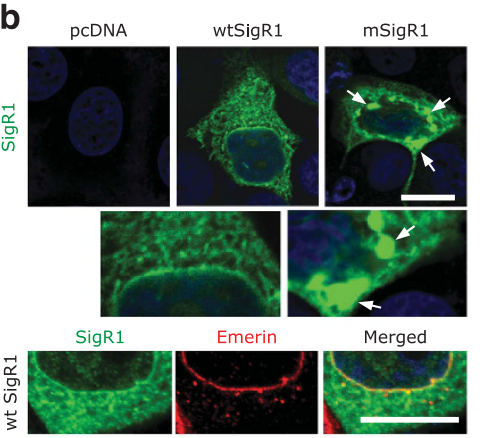

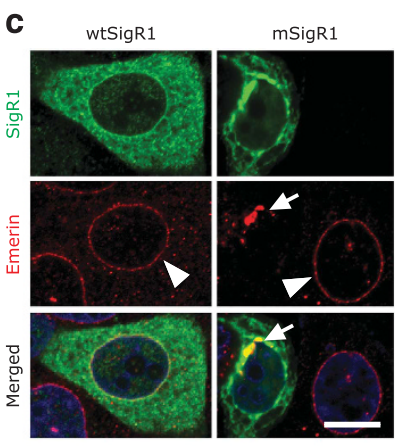

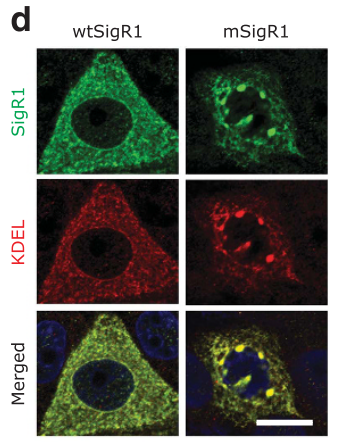

e

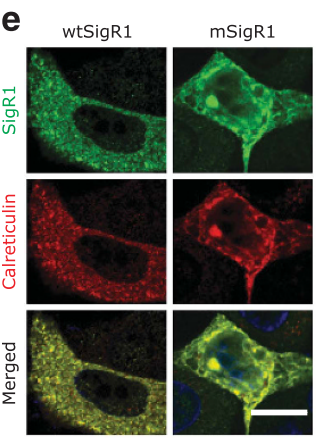

j

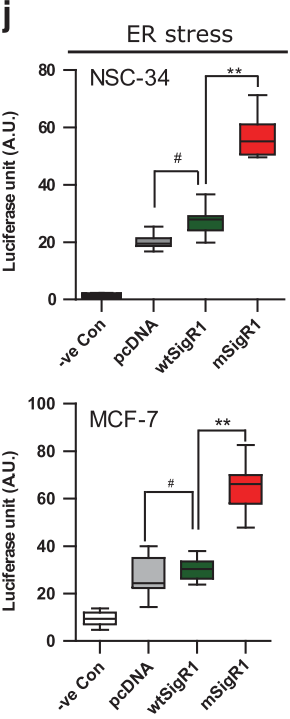

o

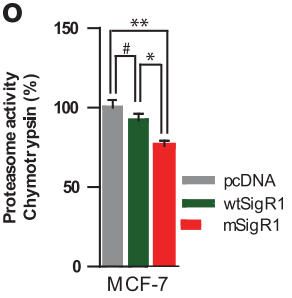

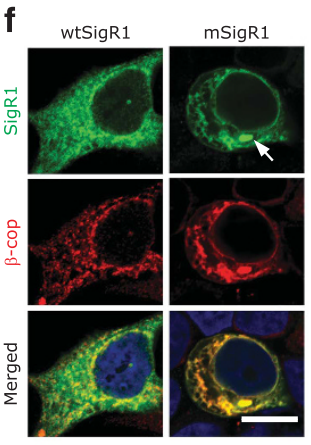
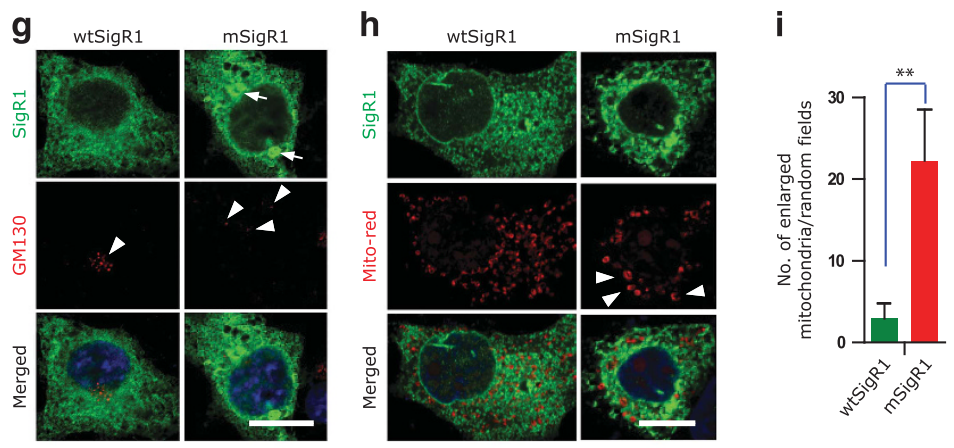

$\mathbf{k}$
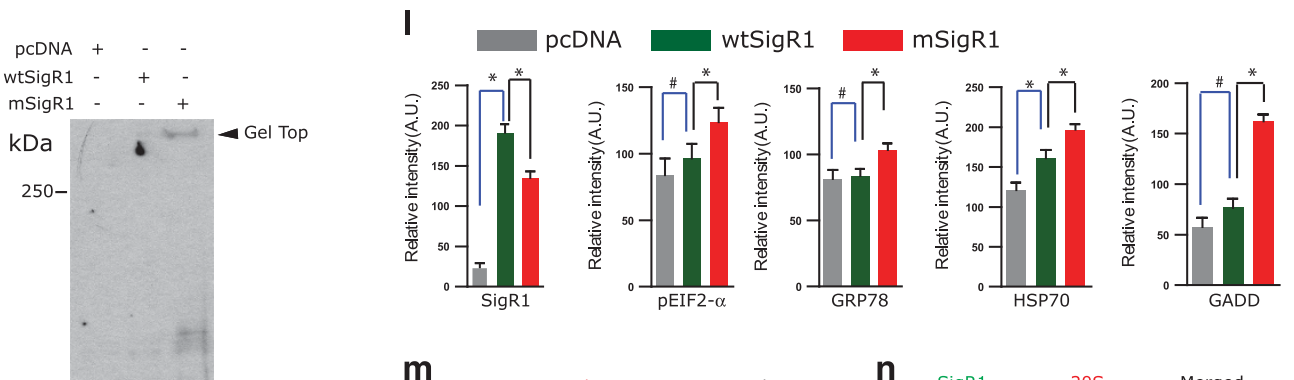

m
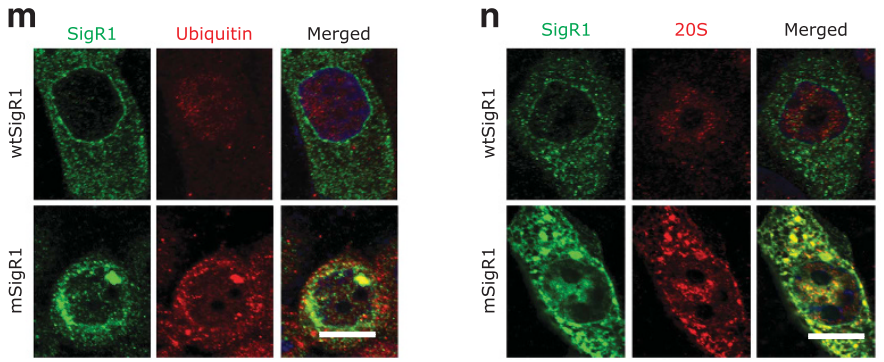
autophagy defects and ER stress-mediated neuronal death. ${ }^{11,14}$ Furthermore, a SigR1 agonist improved motor function and MN survival in SOD1 mice. ${ }^{15}$ These studies suggest a crucial role of SigR1 for neuronal survival. However, it is still unclear whether E102Q-SigR1 causes ALS due to a gain or loss of functions.

Using PLCs obtained from E102Q-SigR1 fALS patients ${ }^{7}$ and autopsy specimens from sALS and fALS patients as well as cell culture models, we found that E102Q-SigR1 aggregates induce ER stress with distinct ER and nuclear envelope alterations, impaired $\mathrm{Ca}^{2+}$ homeostasis and autophagy pathways and aberrant extra-nuclear localization of several ALS-associated RBPs. Our results support the notion that mutant $(\mathrm{m})$ SigR1 induces neuronal toxicity by a loss of normal function combined with a toxic gain of abnormal functions.

\section{Results}

mSigR1 aggregation and cellular toxicity. MCF-7 cells normally express little or no SigR1 (Supplementary Figure 1A). Over-expressed wtSigR1 showed localization in the ER (Figures 1d and e), nuclear envelope (Figures $1 \mathrm{~b}$ and $\mathrm{c}$ ) and ER-Golgi intermediate compartment (Figure 1f) of transiently transfected MCF-7 cells. mSigR1 formed ER-associated aggregates (Figures $1 \mathrm{~b}$ and $\mathrm{d}-\mathrm{f}$ and Supplementary Figures $1 \mathrm{~B}$ and $\mathrm{C}$ ) combined with disintegrated ER membranes and overall cell morphology (Figure 1b,Supplementary Figure 1B). Shuttling of the nuclear envelope protein Emerin was impaired (Figure 1c). These results comply with the recently published crystal structure of SigR1, suggesting that E102Q mutation favors SigR1 aggregation and toxicity. ${ }^{16} \mathrm{mSigR} 1$ aggregates did not associate with the Golgi marker GM130, but there was Golgi dispersal (Figure 1g). SigR1 is abundant at the ERmitochondria-associated membrane (MAM). ${ }^{6}$ Immunoreactivity for the mitochondrial marker Tim23 was reduced, even though few enlarged mitochondria (or clusters of mitochondria) co-localized with SigR1 aggregates (Supplementary Figure $1 \mathrm{H})$. However, consistent with a recent report, ${ }^{9}$ mitochondria overall did not associate significantly with these aggregates, confirmed by mito-red staining (Figures $1 \mathrm{~h}$ and i).

ERSE reporter assay showed increased ER stress in both NSC-34 and MCF-7 cells (Figure 1j) expressing mSigR1. Immunoblotting revealed gel top smear (Figure 1k) and significantly increased levels of the ER stress markers GRP78, pEIF2- $a$, GADD and HSP70 (Figures 1k and I) which correlated well with the expression level of mSigR1 in a dosedependent manner (Supplementary Figures 1D-G). RT-PCR showed a mild, but statistically insignificant increase in RNA of the ER stress markers ATF4 and ATF6 (Supplementary Figures 2A-C), whereas E102Q-SigR1 PCLs showed a significant increase in ATF4, but no ATF6 RNA expression (Supplementary Figure 2D; see below).

Co-localization of Ubiquitin-positive mSigR1 aggregates (Figure $1 \mathrm{~m}$ ) with $20 \mathrm{~S}$ proteasome subunits (Figure 1n) suggest that they were targeted for UPS-mediated degradation and interfered with the UPS machinery. Accordingly, chymotrypsin-like proteasome activity (Figure 10 and Supplementary Figure 1I) was significantly reduced compared to cells expressing empty vector (pcDNA) or wtSigR1.

E102Q-SigR1 fALS patients. We generated immortalized PLCs from blood samples of E102Q-SigR1 fALS patients. ${ }^{7}$ Interestingly, these cells also showed ER-associated mSigR1 aggregates (Figures $2 \mathrm{a}-\mathrm{c}$ ) which co-localized with accumulated Emerin (Figure 2a). mSigR1 aggregates also colocalized with several ER stress markers (Figures 2d and $\mathrm{e}$ ). Dot blot and immunoblot analysis revealed triton- $X$ insoluble gel top aggregates and increased levels of GRP78 and pEIF2- $a$ (Figures $2 f$ and $g$ ). Elevated levels of ubiquitin conjugates, HSP70 and GADD further indicated proteotoxic stress (Figures $2 f$ and $g$ ). Accordingly, both PLCs showed significantly elevated ATF4 mRNA expression (Figure $2 \mathrm{~h}$ and Supplementary Figure 2D). mRNAs of other UPR branches (ATF6, XBP1) remained unchanged (Figure $2 \mathrm{~h}$ and Supplementary Figure 2D). Most importantly, SigR1 mRNA expression showed no significant difference between E102QSigR1 and control PLCs (Figure 2i).

Figure $1 \mathrm{mSigR} 1$ abnormally accumulates in the ER and induces cellular toxicity. (a) Model of the homotrimeric SigR1 (based on ref. 16). Subunits are represented in gray, secondary structure of one subunit is color-coded; each monomer is composed of an amino-terminal transmembrane domain (red) which crosses the ER membrane from lumen to cytosol. The transmembrane domain is followed by two $\alpha$-helices (green) that lead to a $\beta$-barrel (light violet), the putative ligand binding domain. Two carboxy-terminal $\alpha$-helices (blue) form a hydrophobic membrane-embedded surface. The location of the critical residue Glu-102 (E102Q) is marked by a red dot. Red star and red triangle represent two recently discovered mutations. ${ }^{9}$ (b) Reticular pattern of SigR1 staining combined with a distinct nuclear envelope localization (lower panel) in wtSigR1-transfected MCF-7 cells; globular mSigR1 aggregates (arrows; see also enlargement, middle row) in MCF-7 cells expressing mSigR1. Immunofluorescence; scale bars, $15 \mu \mathrm{m}$. (c) Co-immunofluorescence of wtSigR1 and mSigR1 with emerin as a nuclear envelope marker in MCF-7 cells (arrowheads). Note that the focal emerin accumulations (arrows) co-localize with SigR1 aggregates. Scale bar, $10 \mu \mathrm{m}$. (d and e) Co-localization of wtSigR1 and mSigR1 with the ER markers KDEL and (e) calreticulin in MCF-7 cells. Scale bar, $15 \mu \mathrm{m}$. (f and $\mathbf{g}$ ) Co-labelling of SigR1 with the $\beta$-cop (ER-Golgi-associated compartments) and GM130 (Golgi marker). Note the co-localization of mSigR1 with $\beta$-cop and the Golgi dispersal (arrowheads) in mSigR1 expressing MCF-7 cells. Scale bar, $10 \mu \mathrm{m}$. (h) Co-immunofluorescence of wtSigR1 and mSigR1 with mito-red as a marker for mitochondria in MCF-7 cells. Scale bar, $10 \mu \mathrm{m}$. (i) Quantification of the mito-red staining depicted in (h) showing numbers of enlarged mitochondria/random field of view. (j) NSC34 and MCF-7 cells were co-transfected either with empty pcDNA vector, wtSigR1 or mSigR1 together with the luciferase gene downstream of the ERSE promoter. After $48 \mathrm{~h}$, luciferase units (AU) were analyzed as a measure of ER stress. ${ }^{*} P<0.05,{ }^{* *} P<0.005$, \#not significant. (k) MCF-7 cells were transfected with pcDNA, wtSigR1 or mSigR1 as described above and analyzed for ER stress and UPR induction by immunoblotting. (I) Quantification of the band intensities normalized with $\alpha$-tubulin depicted in $\mathbf{k}$. Values represent the mean \pm S.D. of three independent experiments. ${ }^{*} P<0.05$. (m) Ubiquitin immunoreactivity of wtSigR1 and mSigR1 in MCF-7 cells. Scale bar, $10 \mu \mathrm{m}$. (n) Colocalization of mSigR1 aggregates with the accumulated 20s subunit of the proteasome in MCF-7 cells expressing mSigR1. Scale bar, $10 \mu \mathrm{m}$. (o) Chymotrypsin-like proteasomal activity assays were performed using MCF-7 cells transfected with pcDNA, wtSigR1 and mSigR1, as described in material and methods. Note the significant decrease in proteasome activity due to $\mathrm{mSigR} 1$ expression when compared to wtSigR1 and pcDNA. Means \pm S.D. of three independent experiments each performed in triplicate. ${ }^{*} P<0.05$, ${ }^{* *} P<0.005$, \# not significant 
a

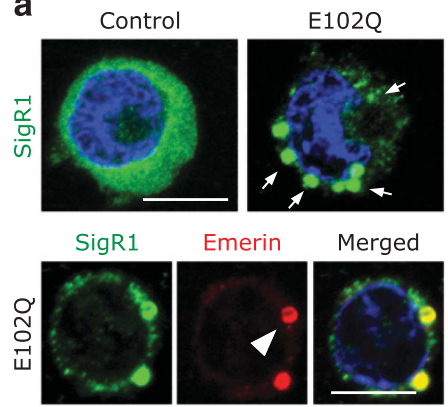

f b

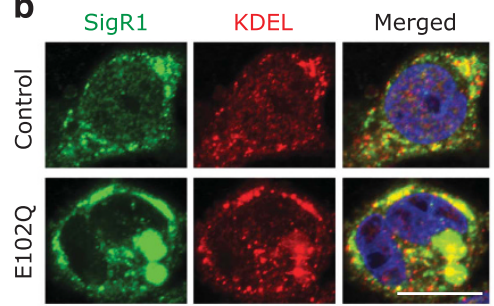

d
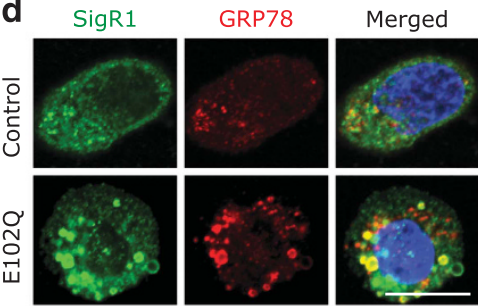

g
Controls E102Q
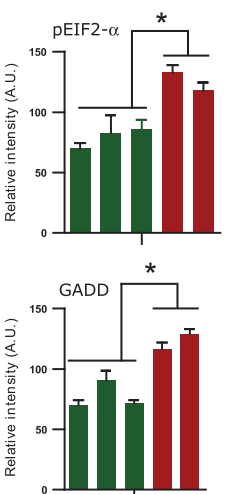
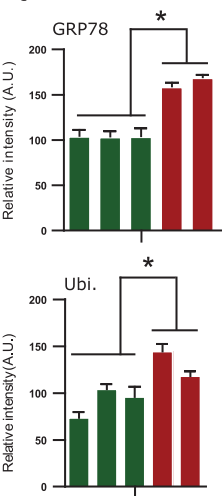

C

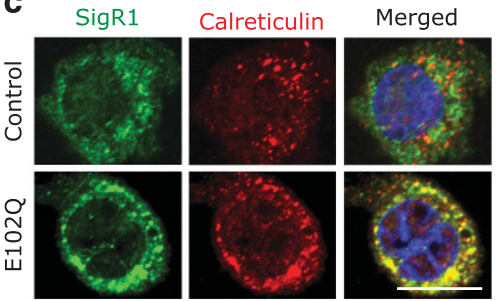

e
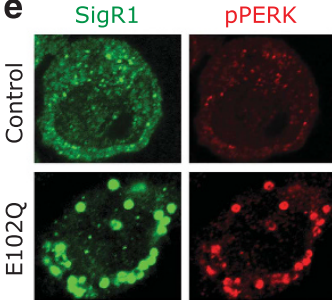

Merged

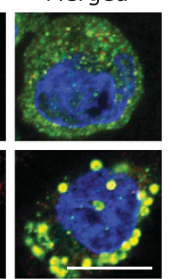

h

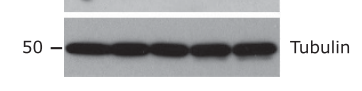

i

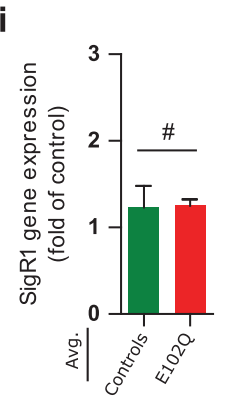

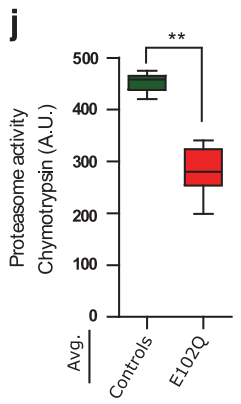

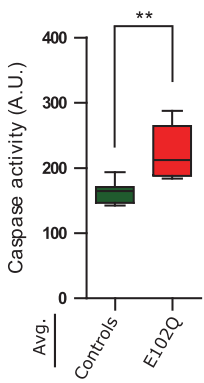

$\mathbf{k}$
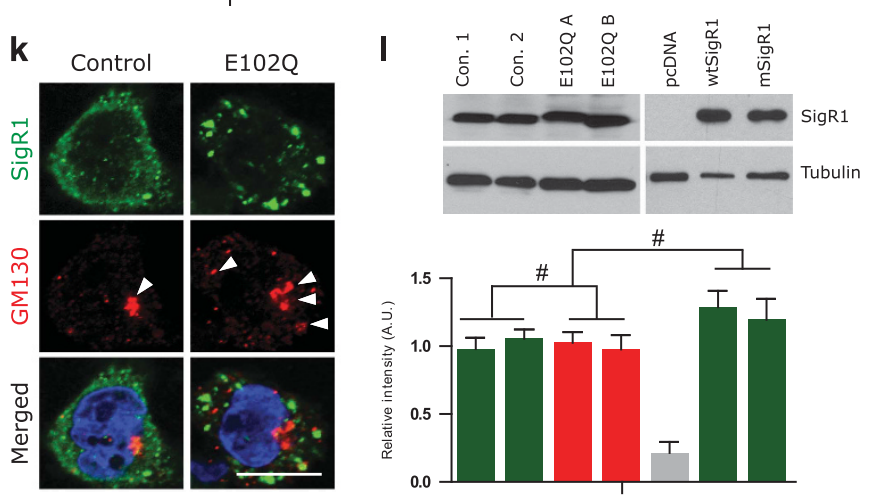

Figure $2 \mathrm{mSigR} 1$ is abnormally accumulated in the ER and induces cellular toxicity in E102Q-SigR1 fALS patient lymphoblastoid cells. (a) Immunoreactivity of globular SigR1 aggregates (arrows) in E102Q-SigR1 fALS patient lymphoblastoid cells compared to the healthy control. Note the co-localization of SigR1 aggregates with the nuclear envelope marker emerin (arrowhead). Scale bar, $15 \mu \mathrm{m}$. (b and c) Co-localization of mSigR1 aggregates with the ER markers KDEL and (c) calreticulin in E102Q-SigR1 fALS patient lymphoblastoid cells compared to the healthy control. Scale bars, $15 \mu \mathrm{m}$. (d and e) Upregulation and co-localization of the ER stress markers GRP78 (d) and pPERK (e) with mSigR1 aggregates in E102Q-SigR1 fALS patient lymphoblastoid cells compared to the healthy control. Scale bars, $15 \mu \mathrm{m}$. (f) Immunoblot analysis of SigR1 and established ER stress and UPR markers in healthy control and E102Q-SigR1 fALS lymphoblastoid cell lysates. Dot blot analysis (top) shows the presence of triton-X insoluble aggregates in E102Q-SigR1 fALS lymphoblastoid cells. (g) Quantification of the band intensities normalized with $\alpha$-tubulin depicted in $\mathrm{f}$. Values represent the mean \pm S.D. of three independent experiments. ${ }^{*} P<0.05$. (h-i) RT-PCR analysis of the UPR pathways in three healthy control lymphoblastoid cell lines compared to two E102Q-SigR1 fALS patient lymphoblastoid cell lines. E102Q-SigR1 fALS patient's lymphoblastoid cells showed a significant increase in ATF4 mRNA expression. * $P<0.05$, \# not significant. Note that SigR1 gene expression does not differ between controls and E102Q-SigR1 fALS patients. (j) E102Q-SigR1 fALS patient's lymphoblastoid cell $(n=2)$ and control lymphoblastoid cell ( $n=3$ ) lysates were subjected to chymotrypsin-like proteasomal activity and caspase-3 activity assays as described in material and methods. Values are derived from the average of three control lymphoblastoid cell lines compared to average of two E102Q-SigR1 fALS patient's lymphoblastoid cell lines from three independent experiment, ${ }^{* *} P<0.005$. (k) GM130 and SigR1 immunolabelling in E102Q-SigR1 fALS and control lymphoblastoid cells. Scale bar, $15 \mu \mathrm{m}$. (I) Immunoblot analysis using SigR1 antibody to compare SigR1 levels in lymphoblastoid cells from E102Q-SigR1 patient lymphoblasts and healthy controls as well as transiently transfected MCF-7 cells. Quantification of the band intensities normalized with $\alpha$-tubulin is shown below. (\#) denotes absence of significant differences 
Decreased proteasomal and increased caspase-3 activity was consistent with these observations (Figure 2j and Supplementary Figure 2E). GM130 staining revealed significant Golgi dispersal compared to the controls (Figure 2k). mSigR1 aggregates did not co-localize significantly with mitochondria (not shown); however, mitochondrial labelling (JC-1 dye) was significantly reduced (see below, Figure 3h). Importantly, SigR1 protein levels actually did not differ significantly between the E102Q-SigR1 PLCs and our transiently transfected cell lines (Figure 2l); the results obtained from the E102Q-SigR1 PLCs and the transiently transfected cell lines were consistent throughout, indicating that the latter were not due to over-expression artefacts.

mSigR1 impairs overall $\mathrm{Ca}^{2+}$ homeostasis and induces mitochondrial toxicity. SigR1 is abundant at the MAM and regulates ER-mitochondria $\mathrm{Ca}^{2+}$ transmission. ${ }^{6}$ mSigR1 induces mitochondrial toxicity ${ }^{17}$ and disturbs ER-mitochondria calcium exchange. ${ }^{9}$ During ER stress and ER $\mathrm{Ca}^{2+}$ deprivation, SigR1 translocates to the plasma membrane to regulate store-operated $\mathrm{Ca}^{2+}$ entry (SOCE) together with channel proteins like STIM1 and Orai1. ${ }^{18}$ Expression of wtSigR1 in our MCF-7 cells efficiently induced IP3Rmediated $\mathrm{Ca}^{2+}$ release from the ER (Figures $3 a$ and $b$ ). mSigR1, however, failed to trigger efficient $\mathrm{Ca}^{2+}$ release (Figures $3 a$ and b), confirming a recent report. ${ }^{9}$ Ionomycin treatment to activate SOCE (Figure 3c) led to significantly increased intracellular $\mathrm{Ca}^{2+}$ in cells transfected with wtSigR1, but not with mSigR1 (Figure 3c). Accordingly, STIM1 protein levels were significantly reduced in MCF-7 cells expressing mSigR1 and in E102Q-SigR1 PLCs (Figures 3d and e).

$\mathrm{Ca}^{2+}$ homeostasis and mitochondrial functions are closely linked. Using the sensitive Tox glow assay we observed significantly impaired mitochondrial membrane integrity (MI) and decreased ATP production in mSigR1-transfected cells (Figure 3f). Consistent with the reduced number of (albeit enlarged) mitochondria (Figure 1h) and reduced Tim23 immunoreactivity in mSigR1-expressing cells (Supplementary Figure $1 \mathrm{H}$ ), the mitochondrial membrane potential was significantly decreased (disappearance of the red-dotted color in the JC-1 stain) in both mSigR1-expressing cells (Figure $3 \mathrm{~g}$ ) and E102Q-SigR1 PLCs (Figure 3h). This led to Cyt-C release (reduced Cyt-C staining, Figure 3i; arrowheads) in mSigR1transfected HeLa and MCF-7 cells.

Structural ER and mitochondria alterations and accumulation of autophagic material. EM revealed numerous vacuoles in mSigR1-expressing cells (Figure $4 \mathrm{a}$ and Supplementary Figure 3A) many of which were probably ER-derived and might actually be precursors of autophagic vacuoles, ${ }^{19}$ often containing non-degraded autophagic substrates (Figure 4a and Supplementary Figure 3A). There was prominent ER swelling (Figure 4b, arrows) compared to the pcDNA control and wtSigR1 over-expressing cells. Higher magnification revealed several autophagic vacuoles with characteristic double membranes and sequestered cellular components in mSigR1-transfected cells (Figure 4c and Supplementary Figure 3B; arrowheads). Furthermore, expression of mSigR1 induced ER widening at the MAM (Supplementary Figure $3 \mathrm{C}$ and Figure 4c, arrow) and led to abnormalities of mitochondrial morphology (swelling of mitochondria, mitophagy) (Figure 4d, arrowheads). In contrast, wtSigR1 over-expressing cells showed rather normal ER, mitochondria and MAM (Figure $4 \mathrm{~b}$ and Supplementary Figure 3C; arrows). Consistent with the nuclear envelope defects seen previously, expression of mSigR1 induced prominent outfoldings of the nuclear envelope (Supplementary Figure 3D). EM of E102Q-SigR1 PLCs revealed similar ultrastructural defects (Figures $4 \mathrm{e}-\mathrm{h}$ ); control PLCs showed rather normal ER, mitochondria and MAM (Figure 4g).

mSigR1 induces defective autophagy. Autophagy largely relies on intact ER structure and functions. We showed morphological ER alterations, disturbed intracellular $\mathrm{Ca}^{2+}$ homeostasis and accumulations of autophagic vacuoles in both mSigR1-expressing cells and in E102Q-SigR1 PLCs; thus we hypothesized that mSigR1 might impair autophagic processes. Autophagy can be monitored at different stages by several well-established methods. ${ }^{14,20,21}$

Transfection of mSigR1 in A431 and MCF-7 cells resulted in increased accumulation of p62 and LC3II (Figure 5a and Supplementary Figure 4A) together with epidermal growth factor receptor (EGFR) and LAMP-1 (Figure 5a). Consistent with the immunoblot results, immunofluorescence (Figure $5 \mathrm{~b}$ ) and CYTO-ID dye labelling (Figure 5c) showed globular LC3 accumulation co-localized with SigR1 aggregates (arrows) in mSigR1-transfected cells. Similarly, p62 was accumulated and abnormally associated with SigR1 aggregates (Figure 5d). Moreover, in nutrient-starved A431 cells (to induce autophagy), expression of $\mathrm{mSigR} 1$ increased the stability of p62 and LC3II levels (Supplementary Figure 4B). Furthermore, autophagy induction (Rapamycin) or inhibition (Bafilomycin A) only marginally, if at all, affected p62 and LC3II levels in mSigR1-expressing MCF-7 cells compared to wtSigR1 controls (Supplementary Figure 4C), suggesting an impairment of autophagic degradation.

mSigR1 expression in mouse fibroblasts (NIH-3T3) stably expressing GFP-LC3 ${ }^{14}$ led to aggregation and abnormal accumulation of GFP-LC3 (Figure $5 \mathrm{e}$ ). Absence of a significant increase in LC3II and p62 levels even after Bafilomycin-A treatment also suggested abnormal autophagy (Figure 5f). In primary MEFs isolated from GFP-LC3 $\mathrm{tg}$ mice $^{22}$ that we transfected with wtSigR1, normal ER-associated SigR1 staining and few GFP-LC3 puncta together with very few p62positive puncta (Figures $5 \mathrm{~g}$ and $\mathrm{h}$ ) were observed. In contrast, mSigR1-transfected MEFs showed sub-plasmalemmal accumulation of mSigR1 (Figure $5 \mathrm{~g}$ ) together with significant globular accumulation and co-localization of GFP-LC3 and p62 (Figure 5h). These findings were corroborated by immunoblot analyses (Supplementary Figure 4D). Finally E102Q-SigR1 PLCs showed similar aggregation and colocalization of p62 and LC3II together with mSigR1 aggregates (Figure 5i), but no co-localization of LAMP1 and SigR1 aggregates (Supplementary Figure 4F). These findings were strengthened by Cyto-ID-LC3 labelling yielding large globular accumulations of LC3 in E102Q-SigR1 PLCs (Figure 5j) and by p62, LC3 and LAMP1 immunoblots (Figure 5k).

Next, we determined which steps within the autophagic processing chain (internalization, fusion or lysosomal degradation) were impaired by mSigR1. Over-expression of mSigR1 
in A431 cells did not impair receptor internalization after 10 min of EGF stimulation compared to wtSigR1 (Supplementary Figure 4E). Activated EGFR was efficiently degraded after internalization in control cells (pcDNA and wtSigR1), however EGFR in mSigR1-expressing cells were only slightly reduced (Figure 5l, quantification below). Live cell imaging of a NIH-3T3 reporter cell line (see Methods and refs 14,23) showed significant impairment of autophagosomes-lysosome a

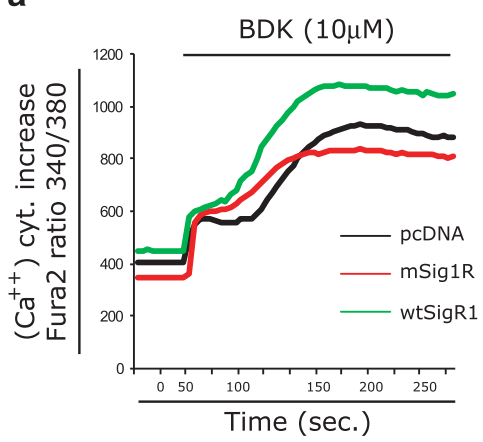

d

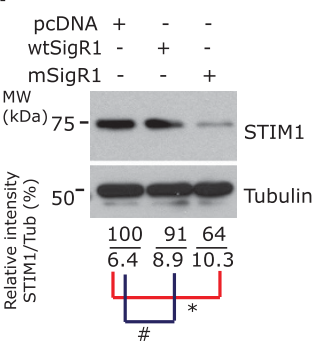

b

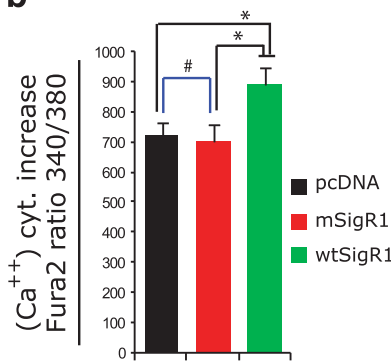

e

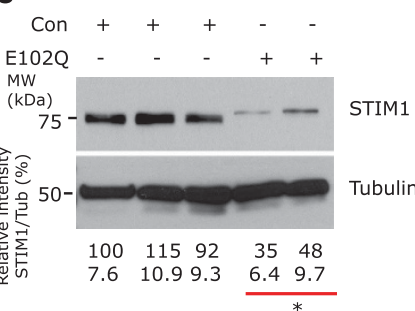

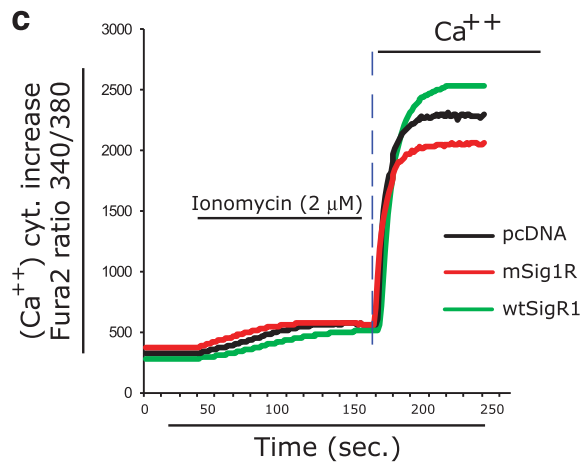

f

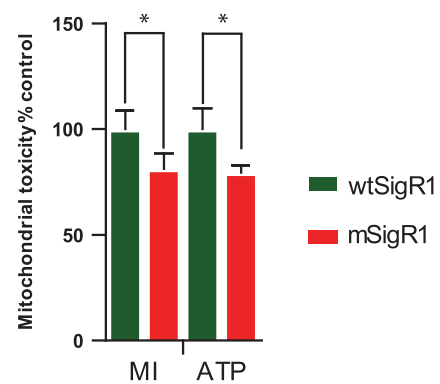

h

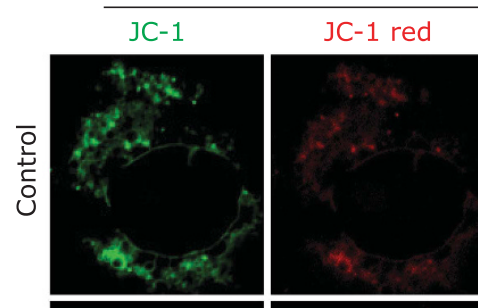

Lymphoblastoid cells

g
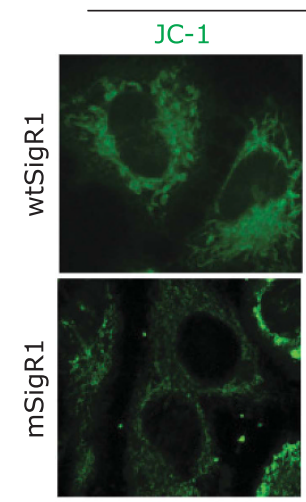

i
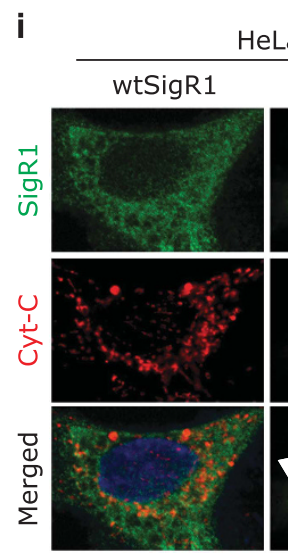

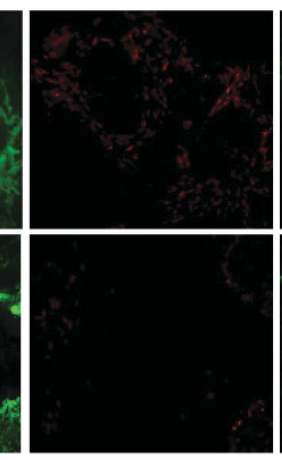

HeLa

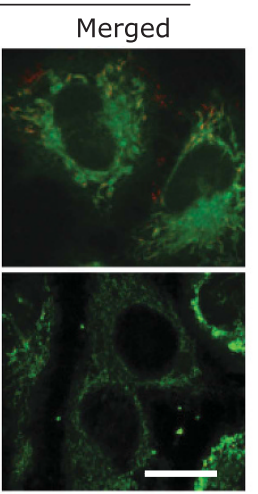

La

mSigR1

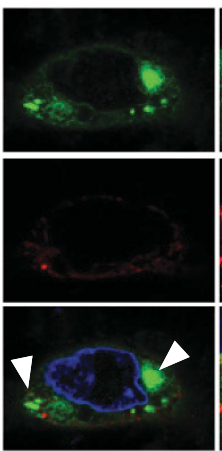

MCF-7

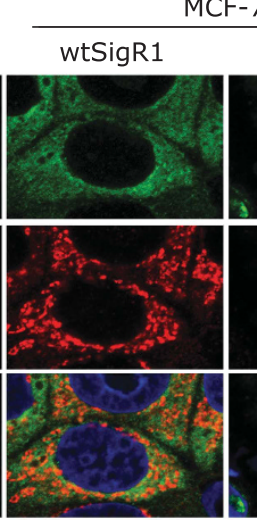

\section{mSigR1}

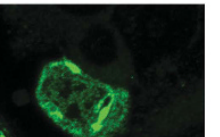

옹
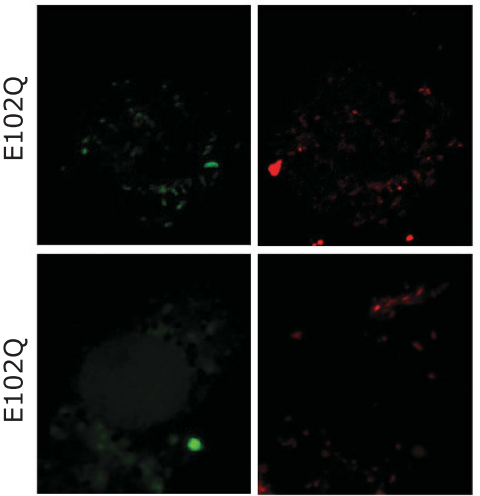

\section{Merged}
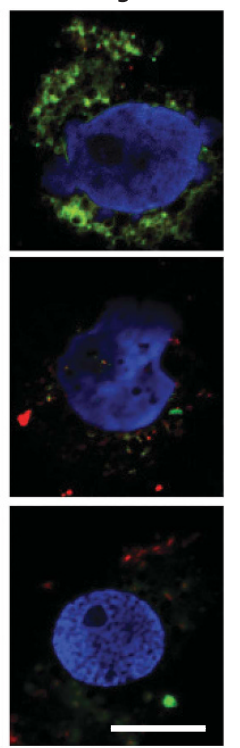
fusion in mSigR1-expressing cells as indicated by the minimal loss of GFP fluorescence and maximal co-localization of RFP and GFP signals (Figure $5 \mathrm{~m}$ ) compared to controls. Accordingly, EM revealed accumulation of double membrane-bound autophagosomes (AV) not fusing with lysosomes (Figure $5 n$ ). Altogether, our findings suggest that mSigR1 aggregates impair the autophagic degradation and clearance of autophagosomes.

mSigR1 impairs vesicular transport. mSigR1 forms ER-associated aggregates (Figures 1a and 6a) accompanied by disturbed ER ultrastructure (Figures $4 a$ and b) and abnormal Golgi morphology (Figures $1 \mathrm{~g}$ and $2 \mathrm{k}$ ). Therefore, ER/Golgi defects might affect vesicular transport from ER to Golgi and vice versa. Thus we analyzed VSVG-GFP fluorescence recovery after photobleaching (FRAP) imaging. $^{24,25}$ In control COS-7 cells co-transfected with VSVG-GFP and either pcDNA or wtSigR1, VSVG-GFP vesicle trafficking was efficient as indicated by fast VSVG-GFP recovery (Figures $6 b$ and $c$ and Supplementary Movie 1). In contrast, in mSigR1-expressing cells intracellular movement of VSVG-GFP vesicles was severely impaired (Figures 6b and $C$ and Supplementary Movie 1). Consistent with this mSigR1 co-aggregated with the endosomal markers Rab5 and Rab7 (Figure 6d) and early endosomal antigen 1 (EEA1) was increased together with ER stress and autophagy markers in immunoblots from mSigR1-transfected COS-7 cells (Figures $6 f$ and $\mathrm{g}$ ). Recapitulating the known effect of over-expression of other ER membrane proteins, ${ }^{26}$ transfection of wtSigR1 mildly affected EEA1 and LC3II, but not p62 and GRP78. E102Q-SigR1 PLCs also showed similar co-aggregation of early (EEA1) and late (Rab7) endosomal markers with mSigR1 (Figure 6e). Our results are consistent with a recent study showing reduced intracellular mobility of mSigR $1,{ }^{27}$ with previous reports describing that VSVG transport is impaired by ER stress, ${ }^{24,25}$ and also with our own previous work showing ultrastructural abnormalities of the ER/Golgi complex in SigR1-deficient cells. ${ }^{11,14}$

mSigR1 accumulation leads to altered distribution of RBPs. RBPs are multifunctional and play a major role in sALS pathophysiology. ${ }^{28}$ Alterations of RBPs homeostasis can directly or indirectly modulate protein quality control. ${ }^{29}$
Cytoplasmic aggregates along with granular cytoplasmic staining for endogenous TDP-43 was found in mSigR1transfected MCF-7 cells, but not in controls (Figure 7a). Similarly cytoplasmic translocation and aggregation of FUS was observed (Figures 7b and d). MEF-GFP-LC3 cells expressing mSigR1 showed increased cytoplasmic staining for endogenous TDP-43 together with increased accumulation of GFP-LC3 (Supplementary Figure 4H).

Mutations in the nuclear matrix protein matrin-3 can cause $\mathrm{fALS}^{30}$ and autosomal dominant distal myopathy with vocal cord and pharyngeal weakness; ${ }^{31,32}$ matrin-3 interacts with TDP-43, FUS and other RBPs. ${ }^{33}$ Matrin-3 also showed cytoplasmic mis-localization in MCF 7 cells expressing mSigR1 (Figures 7c and d). Occasionally in some cells having large globular, compact mSigR1 aggregates, matrin-3 was found exclusively in the cytoplasm with complete loss of nuclear signal (Figure 7c). Immunoblot analysis of subcellular fractions of MCF-7 cells confirmed these observations (Supplementary Figure 4G). Confocal imaging of E102QSigR1 PLCs confirmed that aggregates of mSigR1 were co-localized with TDP-43 (Figure 7e), FUS (Figure 7f) and matrin-3 (Figure 7g). Immunoblots of the subcellular fractions of E102Q-SigR1 PLCs confirmed increased cytoplasmic matrin-3, TDP43 and FUS (Figure 7h). Consistent with these findings immunohistochemistry of the lumbar spinal cord of fALS patients harbouring FUS and C9orf72 mutations revealed cytoplasmic matrin-3 accumulations in $a$-MNs (Figures 7i and j). By double-immunofluorescence these accumulations did not co-localize with pTDP-43 aggregates (Supplementary Figures $5 \mathrm{~A}$ and $\mathrm{B}$ ) consistent with prior observations. ${ }^{30}$ We also confirmed the finding by Johnson et $a l^{30}$ that lumbar $a$-MNs of SALS and fALS patients show increased nuclear matrin-3 immunoreactivity. Interestingly, however, in the E102Q-SigR1 transfected cells and in the E102Q-SigR1 PLCs (Figures 7c and g) a high degree of cytoplasmic SigR1 accumulation was not only associated with increased cytoplasmic matrin-3 immunoreactivity, but also accompanied by a loss of nuclear matrin-3 staining. These data indicate that cytoplasmic mis-localization of matrin-3 in sALS and fALS MNs and in E102Q-SigR1 expressing cells might be pathophysiologically relevant.

Aggregation of these RBPs proceeds through the stress granule pathway (see above and refs 5,34 ). Consistent with

Figure $3 \mathrm{mSigR} 1$ induces mitochondrial toxicity and fails to mobilize IP3R and SOCE-mediated $\mathrm{Ca}^{2+}$ signalling. (a and $\left.\mathbf{b}\right)$ MCF-7 cells were transiently transfected as previously described; $48 \mathrm{~h}$ later cells were loaded with Fura-2AM for $30 \mathrm{~min}$, washed twice and then stimulated with $10 \mu \mathrm{M} \mathrm{BDK}$ under Ca ${ }^{2+}$-free conditions. Average traces of the BDK-induced increase in $\left[\mathrm{Ca}^{2+}\right]^{i}$ from the ER store through IP3R are represented. Note the increase of $\left[\mathrm{Ca}^{2+}\right]^{i}$ in wtSigR1-transfected cells (green curve) compared to a significant decrease in mSigR1-transfected cells (red curve). Results are expressed as mean \pm S.E.M. of $\sim 30$ cells. Mean changes in peak [Ca $\left.{ }^{2+}\right]^{i}$ measured are given. The asterisks denote a statistically significant difference $\left({ }^{*} P<0.05\right)$. (c) Representative SOCE elicited in transfected cells loaded with Fura-2AM as described above. SOCE was triggered by the addition of $2 \mu \mathrm{M}$ ionomycin under $\mathrm{Ca}^{2+}$-free conditions. SOCE developed (vertical dot line) after the addition of $\mathrm{Ca}^{2+}$ is depicted. (d) STIM1 immunoblot analysis from lysates obtained from pcDNA, wtSigR1 and mSigR1-transfected MCF-7 cells. Note the significant down-regulation of STIM1 in mSigR1-transfected cells. The fold change below represents the quantification of band intensities normalized against $\alpha$-tubulin. Values derived from three independent experiments. ${ }^{*} P<0.05$. (e) Significantly decreased STIM1 levels in E102Q-SigR1 fALS lymphoblastoid cell lysates compared to healthy control lymphoblastoid cells. The fold change below represents the quantification of band intensities normalized against $\alpha$-tubulin. Values derived from three independent experiments. ${ }^{*} P<0.05$. (f) Significantly reduced mitochondrial membrane integrity and ATP production in mSigR1 expressing MCF-7 cells compared to wtSigR1 expressing cells measured by the tox glow assay. Values derived from three independent experiments. ${ }^{*} P<0.05$. (g) JC-1 staining of HeLa cells transfected with wtSigR1 or mSigR1. Note the reduced mitochondrial potential in mSigR1 expressing cells. Scale bar, $10 \mu \mathrm{m}$. (h) JC-1 staining of lymphoblastoid cells obtained from E102Q-SigR1 fALS patients and healthy controls. Note the decreased membrane potential in mSigR1 expressing cells. Scale bar, $10 \mu \mathrm{m}$. (i) Cytochrome C immunolabelling of HeLa and MCF-7 expressing wtSigR1 or mSigR1. Note the Cytochrome C release in cells showing mSigR1 aggregates (arrowheads). Scale bar, $10 \mu \mathrm{m}$ 
a

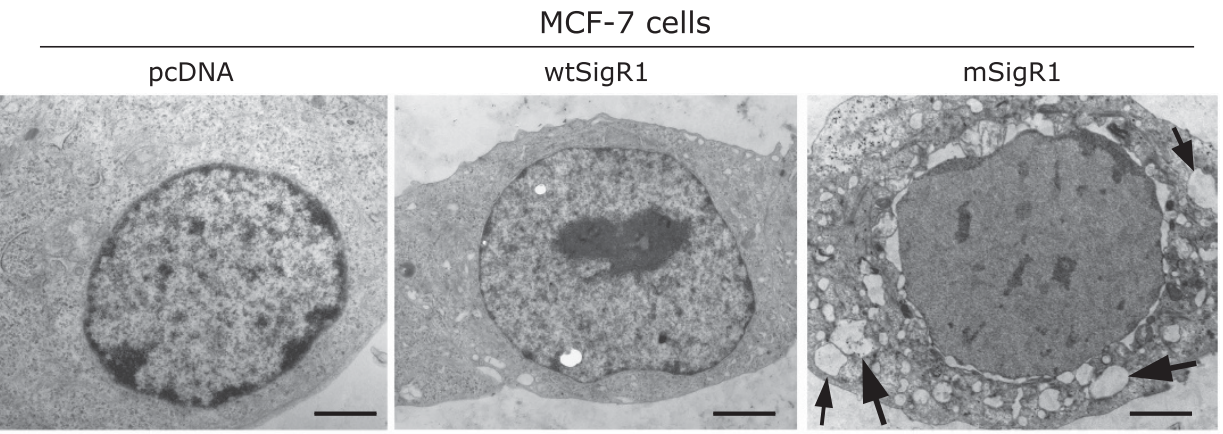

b

pCDNA

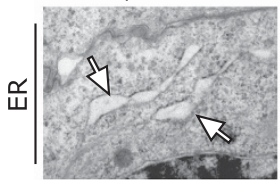

d

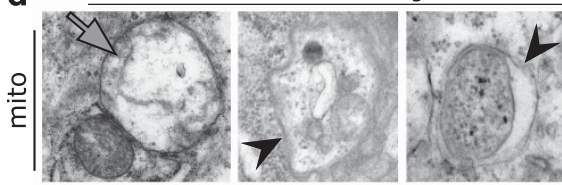

wtSigR1

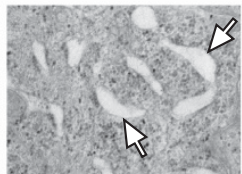

mSigR1
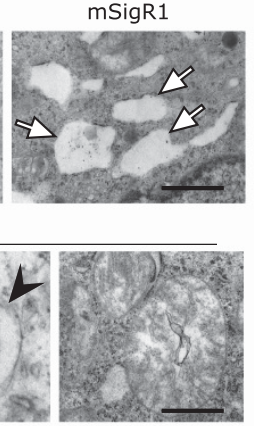

C

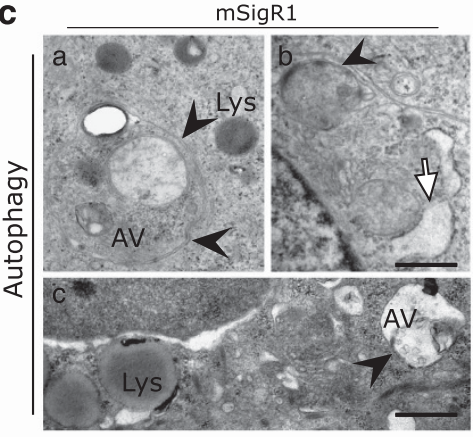

Human lymphoblastoid cells
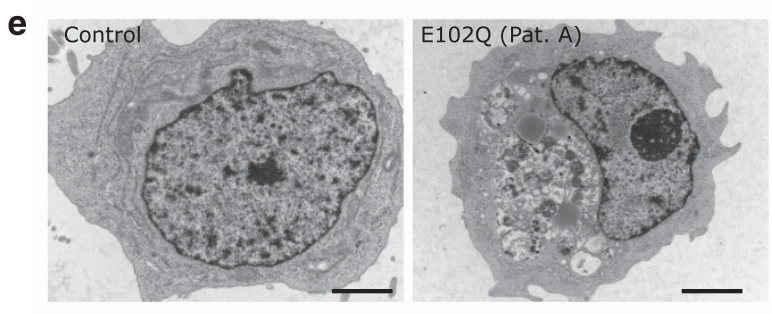

f

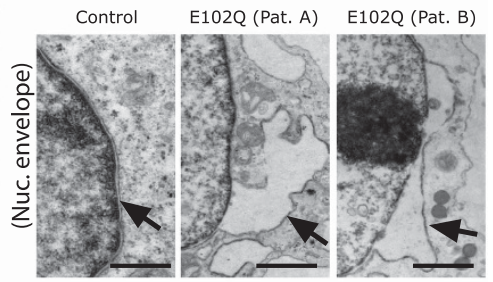

g

Control

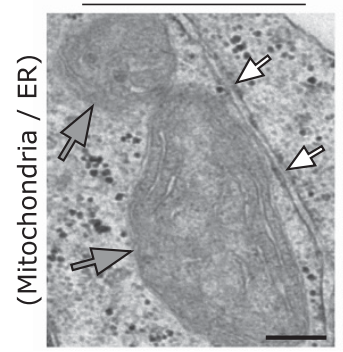

E102Q (Pat. A)

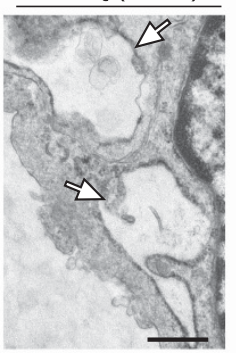

h

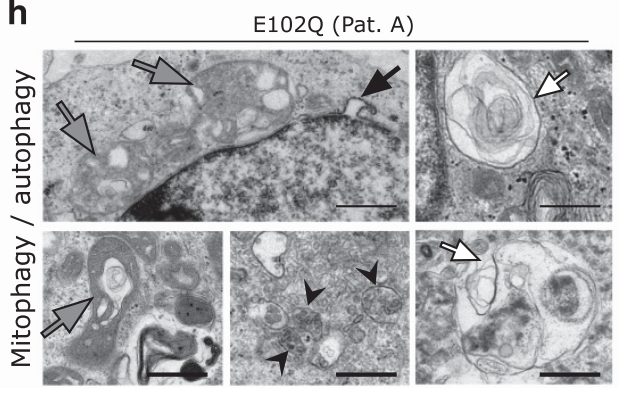

Figure $4 \mathrm{mSigR} 1$ leads to structural abnormalities of ER and mitochondria. (a) MCF7 cells expressing pcDNA, wtSigR1 or mSigR1 were fixed with $2.5 \%$ buffered glutaraldehyde and processed for EM. Several membrane-bound vacuolar structures (black arrows) probably derived from the ER in a representative mSigR1-expressing cell compared to cells expressing wtSigR1 and pcDNA control. Scale bars, $1 \mu \mathrm{m}$. (b) Higher magnification showing the widened ER (arrows in right panel) in representative mSigR1expressing cells compared to pcDNA and wtSigR1-transfected cells. Scale bar, $0.5 \mu \mathrm{m}$. (c) Higher magnification showing double membrane autophagic vacuoles in representative mSigR1-expressing MCF-7 cells. (a) Large autophagosome (AV; arrowheads) containing mitochondria and other structures in close proximity to lysosomes (Lys); (b) autophagosome (arrowhead) and widened ER (white arrow); (c) autophagosome (AV) and lysosomes (Lys). Scale bar, $0.5 \mu \mathrm{m}$. (d) Enlarged mitochondria (arrows) showing abnormal cristae architecture, some undergoing mitophagy (arrowheads) in mSigR1-transfected cells. Scale bar, $0.4 \mu \mathrm{m}$. (e) Primary lymphoblastoid cells from healthy control and E102Q-SigR1 fALS patients were fixed with $2.5 \%$ buffered glutaraldehyde and processed for EM. Control lymphoblasts show an overall normal ultrastructure, whereas E102Q-SigR1 fALS patient lymphoblastoid cells reveal prominent accumulation of autophagic material. Scale bars, $2.5 \mu \mathrm{m}$. (f) Prominent nuclear envelope (arrows) protrusions in E102Q-SigR1 fALS lymphoblastoid cells and rather normal nuclear envelope in control lymphoblastoid cells. Scale bar, $0.5 \mu \mathrm{m}$. (g) Normal ER (white arrows) and mitochondria (gray arrows) in healthy control lymphoblastoid cells; E102Q-SigR1 fALS patient lymphoblastoid cells showing overall widened ER (arrows). Scale bar, $0.5 \mu \mathrm{m}$. (h) E102Q-SigR1 fALS lymphoblasts displaying vacuolar degeneration of mitochondria (gray arrows), multivesicular bodies (arrowheads) and autophagosomes filled with membranous and granular material (white arrows). Black arrow: nuclear envelope protrusion. Scale bars, $0.4 \mu \mathrm{m}$ 
the cytoplasmic mis-localization of several RBPs, MCF-7 cells expressing mSigR1 showed prominent Tia 1-positive stress granules co-localized with SigR1 aggregates (Figures 7k and I) and cytoplasmic P-body formation in both E102Q-SigR1 PLCs and transfected MCF-7 cells (Supplementary Figure $4 \mathrm{I}$ and J). However SigR1 aggregates did not exactly co-localize with adjacent $\mathrm{P}$ bodies (Supplementary Figures $4 \mathrm{I}$ and $\mathrm{J}$ ). These results indicate that mSigR1 expression and aggregation leads to abnormal RNA-binding protein homeostasis and stress granule formation.

E102Q-SigR1 mediated ALS pathogenesis. Schematic representation (Figure 8 ) of the interdependent pathophysiological mechanisms of ALS pathogenesis associated with the E102Q mutation in SigR1.

\section{Discussion}

Mutations in several ER proteins, including SigR1, cause MNDs. For instance, the P56S mutation in the VAPB gene leads to a form of fALS, ALS-8, ${ }^{35,36}$ characterized by distinct ultrastructural ER alterations and defective protein degradation pathways. ${ }^{37}$ Similarly, mutations in ER chaperones such as SIL1, HSPB8 and HSJ1 lead to familial neurodegenerative disorders including MNDs. ${ }^{38-40}$ ER (co-) chaperones including SigR1 and SIL1 accumulate in surviving MNs in SALS and might serve protective functions. ${ }^{11,41} \mathrm{E} 102 \mathrm{Q}-\mathrm{SigR} 1$-associated disease shows an autosomal recessive inheritance pattern suggesting a 'loss-of-function' pathomechanism consistent with a recent report $^{42}$ and also with our previous reports. ${ }^{11,14}$ However, neither the E102Q nor the recently found homozygous (E138Q and E150K) SigR1 mutations ${ }^{9}$ could be linked to transcriptional silencing or defective translation so far.

ER stress and structural alterations of the ER/nuclear envelope. ATF4 is required for the activation of SigR1 transcription and upregulation of SigR1 suppresses ER stress-mediated cell death, thus considered to be neuroprotective. ${ }^{43}$ Consistent with this, Gregianin et al. ${ }^{9}$ recently showed that over-expression of wtSigR1 suppressed ER stress-induced mitochondrial injury. We found that mSigR1 promotes prolonged ER stress (Figures 1j-I and 2d-g) and altered ER/Golgi morphology (Figure 4) followed by defective endosomal trafficking (Figures $6 \mathrm{~b}$ and $\mathrm{c}$ ). We previously observed ER stress and defects in endosomal trafficking after SigR1 protein depletion. ${ }^{11,14}$ Moreover, E102Q-SigR1 was recently shown to lose its ability to bind to the MAM, suggesting yet another loss of function pathomechanism. ${ }^{9}$ Evidence favoring an additional toxic gain of function results from very recent findings suggesting reduced mobility of E102Q-SigR1. ${ }^{27}$ Analogously, the wtVAPB protein is involved in UPR and P56S-mutated VAPB causes ALS-8, ${ }^{35,36}$ forms ER-associated aggregates, alters ER/Golgi ultrastructure, protein quality control ${ }^{37,44}$ and endosomal trafficking ${ }^{45,46}$ and causes ER stress-related cell death in vitro $^{44}$ and motor abnormalities in P56S-VAPB transgenic mice. ${ }^{37}$ Loss of SIL1, another ER (co-)chaperone, affects ER homeostasis in mouse models; ${ }^{47}$ loss of a single functional SIL1 allele in the G93A-SOD ALS mice enhanced ER stress and exacerbated ALS pathology. ${ }^{41}$ These results are reminiscent of the phenotypes in $S i g R 1^{-/-}$mice. ${ }^{13}$ Conversely, AAV delivery of SIL1 to fALS MNs restored ER homeostasis, delayed muscle denervation and prolonged survival $^{41}$ similar to the improved motor function and $\mathrm{MN}$ survival in SOD1 fALS mice after treatment with a SigR1 agonist. ${ }^{15}$

SigR1 mainly resides at the ER-MAM interface; upon agonist-mediated activation SigR1 translocates from the ER to the nuclear envelope where it binds to emerin and recruits chromatin-remodeling molecules including lamin $\mathrm{A} / \mathrm{C}{ }^{48}$ Knockdown of SigR1 protein induces disruption of the nuclear envelope complex. ${ }^{48}$ In line with these observations, we observed reduced emerin staining of the nuclear envelope in E102Q-SigR1-transfected cells (Figure 1b) and E102Q-SigR1 PLCs (Figure 2a). In addition, we found aberrant emerin co-localization with mSigR1 aggregates indicative of an additional toxic gain of function (Figure 1c,Figure 2a). Interestingly, SIL1-deficient mice and human Marinesco Sjögren syndrome (MSS) patients lacking SIL1 also display myonuclear envelope defects. ${ }^{47}$ Consistent with this P56S-VAPB affects the retrograde trafficking of ERGIC- $53^{49}$ and shuttling of emerin from the ER to the inner nuclear membrane, ${ }^{49}$ and knockdown of endogenous VAPB actually recapitulates this phenotype. ${ }^{49}$ Thus, nuclear envelope defects emerge as a common denominator of pathologies associated with the ALS proteins SigR1, VAPB and SIL1.

MAM, mitochondrial impairment and intracellular calcium dysregulation. Deregulation of neuronal intracellular $\mathrm{Ca}^{2+}$ signaling is a major pathomechanism observed in many neurodegenerative disorders such as $A D, P D, A L S$ and $H D$ (reviewed in ref. 50). Besides its chaperone function SigR1 also regulates IP3-mediated $\mathrm{Ca}^{2+}$ homeostasis at the $\mathrm{MAM}^{6}$ and depletion of SigR1 disturbs $\mathrm{Ca}^{2+}$ homeostasis ${ }^{11,14}$ and ER-mitochondria crosstalk. ${ }^{13}$ MAM alterations are involved not only in ALS but also in AD, PD and in several axonopathies such as hereditary spastic paraplegia and axonal Charcot-Marie-Tooth disease (reviewed in ref. 51). In the present study, mSigR1 expression led to MAM alterations and impaired SOCE and failed to trigger efficient $\mathrm{Ca}^{2+}$ release from the ER through IP3R (Figures $3 a-c$ ). These calcium handling and MAM defects are most likely caused by both the observed disruption of $\mathrm{ER} / \mathrm{mitochondrial}$ structure and by alterations in STIM1-mediated $\mathrm{Ca}^{2+}$ influx because of lowered levels of STIM1 in the E102Q-SigR1-expressing cells (Figures $3 d$ and $e$ ). These findings are in line with our previous studies ${ }^{11,14}$ and also with the recent study by Gregianin et al. describing the deleterious effect of two new mutations in SigR1 (E138Q and E150K) on cell viability due to an altered MAM and impaired global $\mathrm{Ca}^{2+}$ signalling. ${ }^{9}$ Interestingly, another study (by Tagashira et al. ${ }^{17}$ ) using transient expression of E102Q-SigR1 showed aberrant mitochondrial $\mathrm{Ca}^{2+}$ uptake and ATP production, ${ }^{17}$ consistent with our results. However, both groups studied only IP3Rmediated $\mathrm{Ca}^{2+}$ homeostasis and its effects on mitochondria. In contrast, we also examined STIM1-induced SOCE as an alternate mechanism causing globally altered $\mathrm{Ca}^{2+}$ homeostasis. 
Proteostasis (UPS/autophagy) and RBPs. The turnover of TDP-43 and FUS and of other RNA stress granule proteins is regulated by UPS and autophagy; ${ }^{52,53}$ chemically augmenting autophagic flux leads to the clearance of abnormal aggregates of these proteins. ${ }^{52,53}$ Furthermore, alterations of mRNA biogenesis/processing can modulate protein expression under various cellular stress responses. ${ }^{29} \mathrm{We}$ found that mSigR1 aggregation leads to ER stress (Figures 1 and 2), autophagy impairment (Figure 5) and aberrant extranuclear localization and aggregation of the RBPs TDP-43, FUS and matrin-3 (Figures 7a-h). Matrin-3 regulates transcription and stability of several RBPs including TDP-43

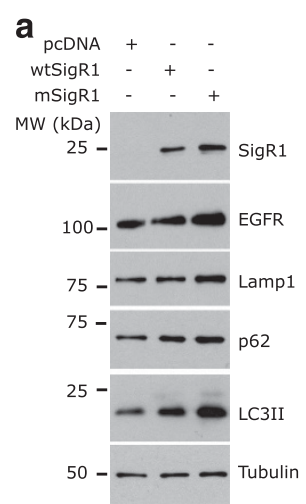

b
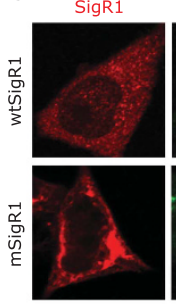

e

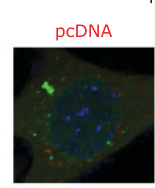

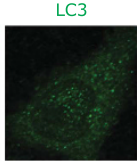

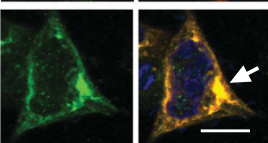

NIH3T3 (GFP-LC3)

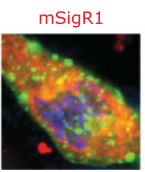

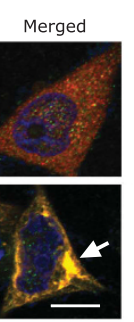

wtSigR1

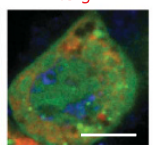

c
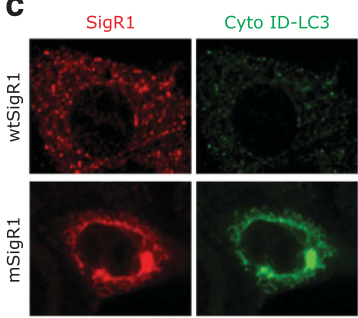

f pCDNA
mSSig1
wtSigR1

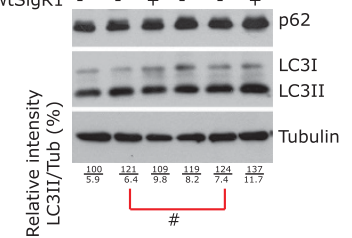

d
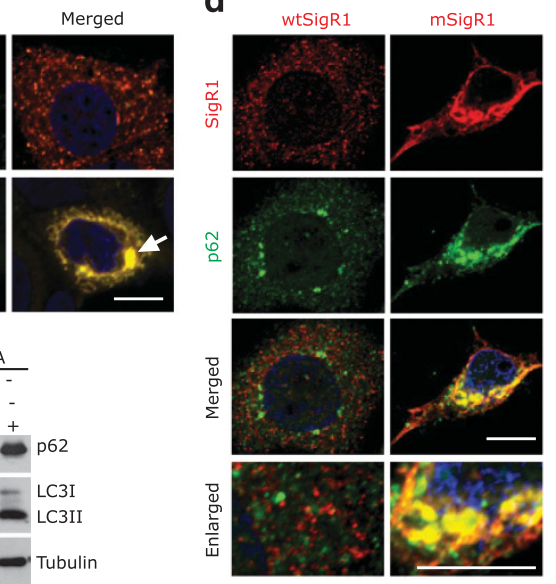

MEF EGFP-LC3 cells

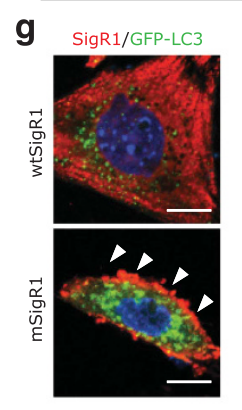

j

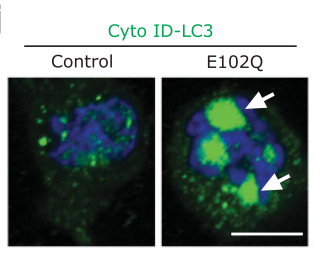

k

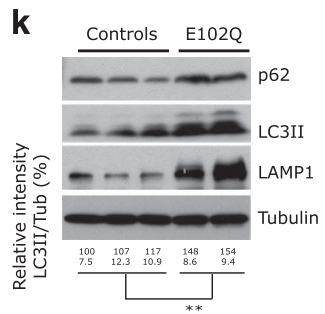

n

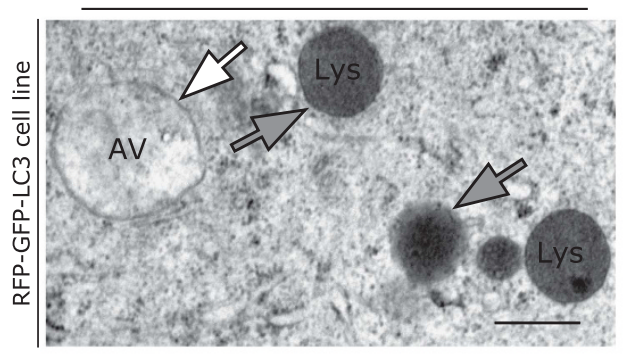

i E102Q Lymphoblastoid cells
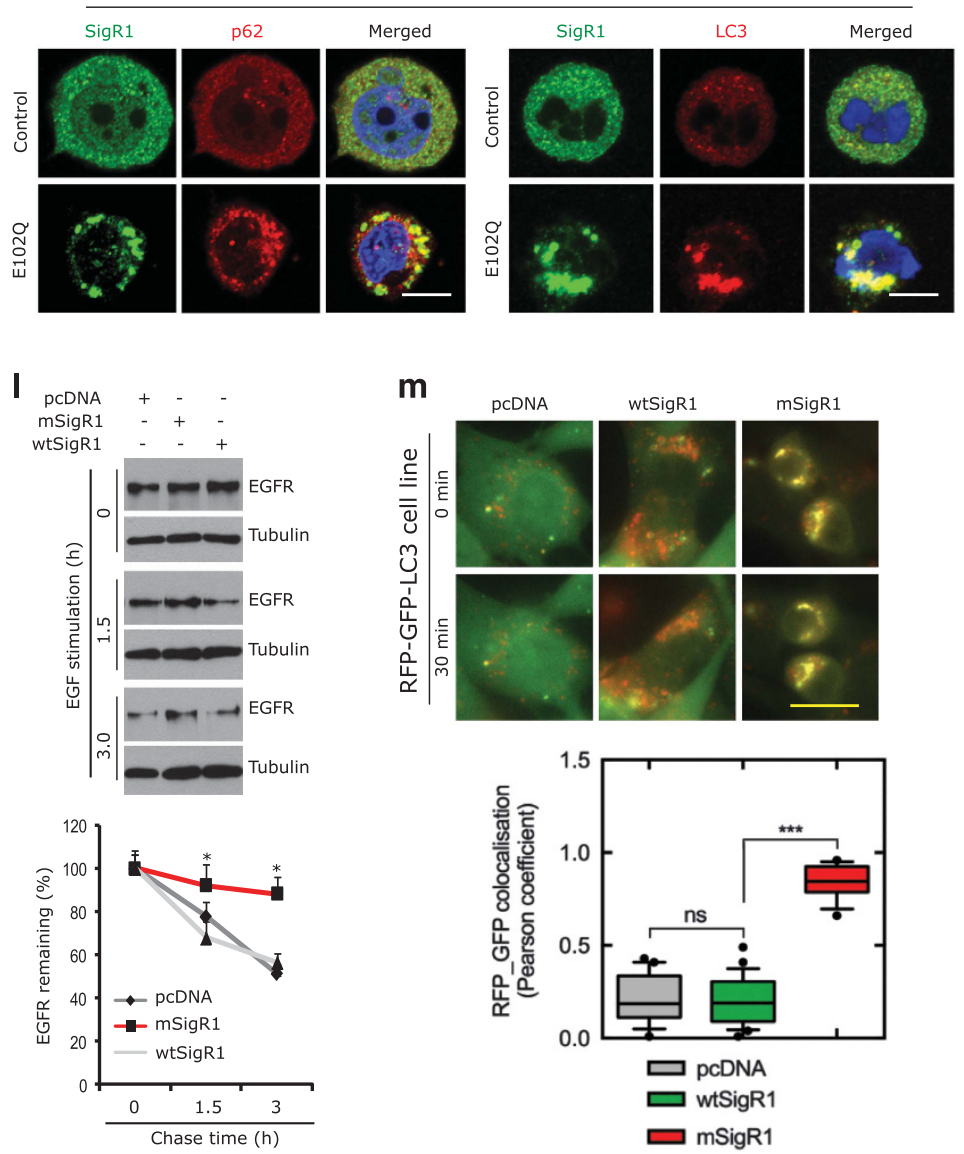
and FUS; ${ }^{33}$ MATR3 mutations cause ALS and distal myopathy. ${ }^{30-32}$ Recently, mice over-expressing human matrin-3 were reported to develop muscular atrophy and altered spinal cord distribution of matrin-3 protein. ${ }^{54}$ Consistent with previous reports ${ }^{30-32}$ on human matrinopathy, we observed both cytoplasmic and nuclear matrin-3 accumulation in E102Q-SigR1 over-expressing cells, along with the aggregation of other RBPs relevant to ALS (TDP-43 and FUS). Furthermore, matrin-3 mis-localization was induced by misfolded protein stress and impairment of degradation pathways in mSigR1 expressing cells (Supplementary Figure 5C). Interestingly, transfected cells showing large cytoplasmic accumulations of SigR1 also showed increased cytoplasmic matrin-3 immunoreactivity suggesting that the E102Q-SigR1 mutation leads to a toxic gain of function involving matrin-3 (Figures $7 \mathrm{c}$ and $\mathrm{g}$ ). These results are consistent with a previous report demonstrating cytoplasmic accumulation of matrin-3 in $a-\mathrm{MNs}$ of ALS patients harboring C9ORF72 and MATR3 mutations. Here, we confirm the increased cytoplasmic matrin-3 immunoreactivity in C9ORF72 fALS $a$-MNs and demonstrate that cytoplasmic matrin-3 aggregation also occurs in lumbar $a$-MNs of fALS patients harboring FUS mutations. Irrespective of the presence or absence of cytoplasmic matrin-3 aggregation, we also confirm the observation that nuclear matrin-3 staining is increased in SALS and fALS lumbar $a$-MNs. ${ }^{30}$ However, unlike mTDP-43 and mFUS, cytoplasmic accumulations of matrin-3 in fALS a-motor neurons are rare. ${ }^{30}$ Taken together, our immunohistochemical data from human ALS autopsy cases are in line with the above described results obtained with E102Q-SigR1 over-expressing cultured cells. Our data indicate that cytoplasmic mis-localization of matrin-3 together with other RBPs in SALS and fALS cells as well as in E102QSigR1 expressing cells might be pathophysiologically relevant.

With regard to TDP-43 redistribution in E102Q-SigR1 transfected cells, our data confirm results of a recent study ${ }^{17}$ describing TDP-43 mis-localization along with deranged mitochondrial ATP production and proteasome activity as a consequence of mSigR1 over-expression. Our observations are also in accordance with studies examining a causal relationship of other ER proteins like Sil1 and VAPB with TDP-43 proteinopathy. P56S-VAPB, for instance, potentiated the TDP-43-induced MN death, whereas wt-VAPB had opposite effects. ${ }^{45}$

We observed that both the ER stressor thapsigargin and the autophagy inhibitor bafilomycin A led to similar extra-nuclear localization of TDP-43 and matrin-3 in NSC-34 (motor neuronlike) cells; induction of autophagy either by rapamycin or by the SigR1 agonist PRE084 prevented this mis-localization (Supplementary Figures 5C and D). SigR1 agonists such as PRE084 have already been shown to reduce protein aggregation and ER stress ${ }^{11}$ and to be neuroprotective. ${ }^{15}$ Thus, our data suggest that the ALS proteins SigR1, TDP-43 and matrin-3 are interdependent in regulating cellular protein quality control pathways and that dysregulation of the RBPs matrin-3, FUS and TDP-43 can be directly caused by mSigR1 aggregation. In addition, indirect mechanisms such as autophagy impairment due to mSigR1 expression could be active, supporting a simultaneous toxic gain and loss of function of mSigR1 (summarized in Figure 8), similar to the recently described pathomechanism (s) related to mutated TDP-43, C9orf72, FUS, ${ }^{46}$ SOD1 (ref. 55) and VAPB. ${ }^{44,49}$

\begin{abstract}
Materials and Methods
Reagents. Fluorescent nucleic acid stain Hoechst 33258 was purchased from Molecular Probes (Eugene, OR, USA). Thapsigargin,Tunicamycin, EGF, Rapamycin, Bafilomycin A, Fura 2AM, $\mathrm{NaCl}, \mathrm{KCl}$, pluronic acid, $\mathrm{CaCl}$, $\mathrm{MgCl}$, glucose, HEPES 4-(2-hydroxyethyl)-1-piperazineethanesulfonic acid, N-acetyl-Asp-Glu-Val-Asp-7 amido-4-methylcoumarin and Bradykinin were purchased from Sigma Aldrich (Munich, Germany). Suc-Leu-Leu-Val-Tyr-AMC was received from Enzo (Lausen, Switzerland). Alamar Blue was purchased from Invitrogen, Thermo Fischer Scientific, Carlsbad, CA, USA. The CYTO-ID Autophagy detection kit was purchased from Enzo.
\end{abstract}

Antibodies. The antibodies used in this study and their dilutions are described in Supplementary Table 1.

Cell culture, transient transfection and treatments

Cell culture and treatment. Human epithelial cancer cells (HeLa), African green monkey kidney cells (COS-7), human breast cancer cells (MCF-7) and NSC34 motor neuron-like cells were cultured in Dulbecco's modified Eagle's

Figure $5 \mathrm{mSigR} 1$ leads to defective autophagy and autophagosome-lysosome fusion. (a) Immunoblot analysis for autophagy markers in A431 cells transiently transfected with pcDNA, wtSigR1 and mSigR1. Note the increased levels of autophagy markers in mSigR1 expressing cells. (b-d) MCF-7 cells were transiently transfected with wtSigR1 or mSigR1 and then processed for (b) co-immunolabelling using SigR1 and LC3 antibodies, (c) co-immunolabelling using SigR1 and Cyto-ID dye (for labelling autophagosomes) and (d) co-immunolabelling using SigR1 and p62 antibodies. Scale, $10 \mu \mathrm{m}$. (e) Increased accumulation of autophagosomes in the stable autophagy reporter cell line NIH3T3GFP transfected with mSigR1. Green: GFP-LC3; red: SigR1; scale bar, $10 \mu \mathrm{m}$. (f) Immunoblot analysis of NIH3T3-GFP-LC3 cells transfected with wtSigR1 or mSigR1 and additionally treated with the autophagy inhibitor Bafilomycin A for $2 \mathrm{~h}$. Note the unchanged LC3-II levels in mSigR1-transfected cells after Bafilomycin A treatment. Corresponding densitometric data are shown at the bottom; where the upper number represents the relative LC3ll/Tub levels and the lower numbers are the S.D. The asterisks $\left.{ }^{*}\right)$ denote significant differences $\left({ }^{*} P<0.05\right)$, while \# denotes absence of a significant difference. ( $\mathbf{g}$ and $\mathbf{h}$ ) Primary fibroblasts isolated from autophagy reporter GFP-LC3 transgenic mice were transfected with wtSigR1 or mSigR1 and immuno-labelled by SigR1 $\mathbf{g})$ and p62 (h) antibody (merge image for GFP-LC3, green, and SigR1/p62, red). Note the localization of SigR1 at the periphery of this particular cell (g, arrowheads) and the co-localization of GFP-LC3 with globular p62 accumulations. Scale bar, $10 \mu \mathrm{m}$. (i) Co-localization of SigR1 with p62 and LC3 in E102Q-SigR1 fALS lymphoblastoid cells compared to healthy control lymphoblastoid cells. (j) Cyto-ID (green) staining (right) showing the accumulation of autophagosomes in E102Q-SigR1 fALS lymphoblastoid cells. (k) Immunoblot analysis of established autophagy markers in E102Q-SigR1 fALS lymphoblastoid cells in comparison to healthy controls. (I) A431 cells were transfected as described above. Forty-eight hours later, transfected cells were processed for the EGFR degradation assay as described in Materials and Methods and analyzed by immunoblotting with the EGFR antibody. Note the delayed EGFR degradation in mSigR1 expressing cells. (lower) Quantification of immunoblot analysis. Values are expressed as mean \pm S.D. from three independent experiments. ${ }^{*} P<0.05$. (m) NIH3T3 cells expressing RFP-GFP-LC3 were transfected with pcDNA, wtSigR1 or mSigR1. Forty-eight hours later the fusion of autophagosomes with lysosomes was measured by live cell imaging. Scale bar, $25 \mu \mathrm{m}$. (lower) The rate of autophagosome maturation reflected by the Pearson coefficient (green/red fluorescence ratio) at each time point indicated. Values are represented as means \pm S.E.M. of triplicate experiments ${ }^{*} P<<0.0001$. (n) EM picture of NIH-3T3 cells stably expressing RFP-GFP-LC3 transfected with mSigR1. Note the autophagosome (white arrow) and lysosomes (gray arrow) without any fusion 
a
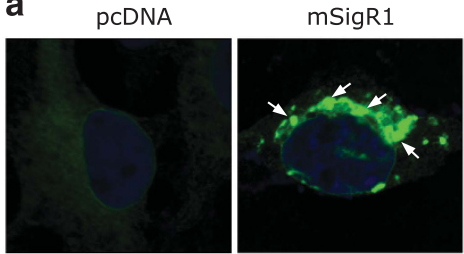

C

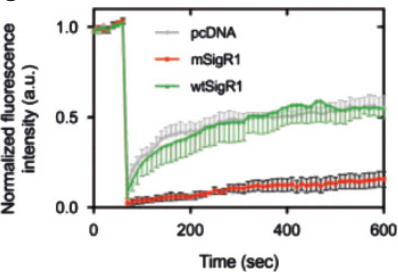

d

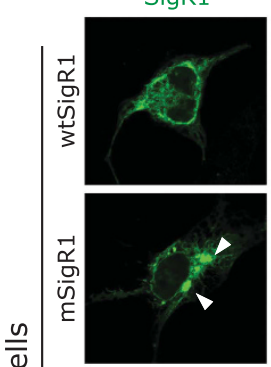

SigR1

仓̊

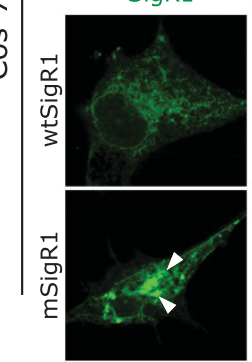

Rab 5
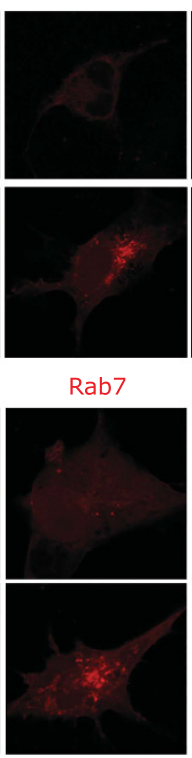

Merged
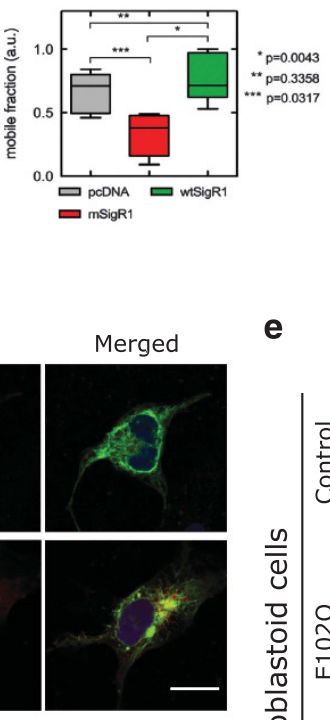

Merged

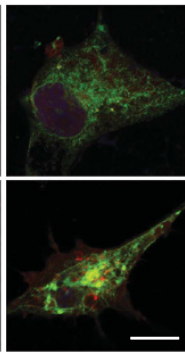

e b
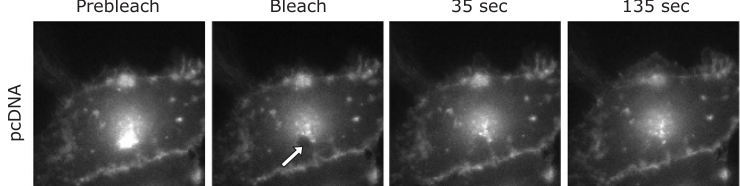

$235 \mathrm{sec}$
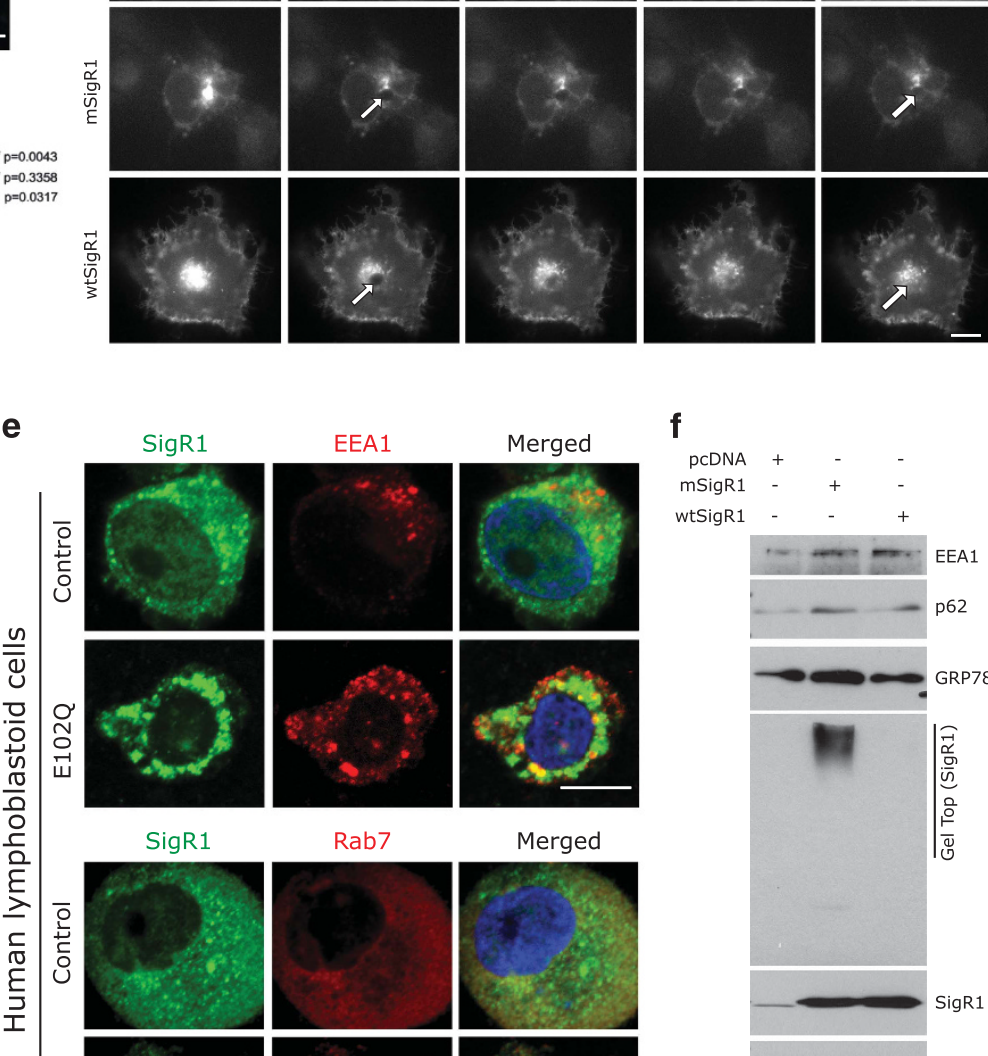

Rab7

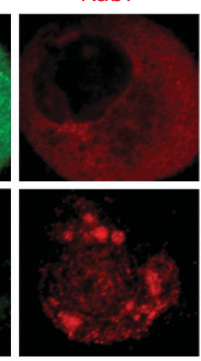

Merged

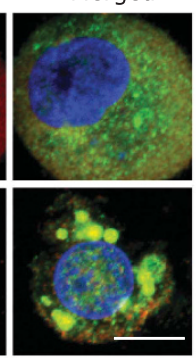

mSigR1 wtSigR1

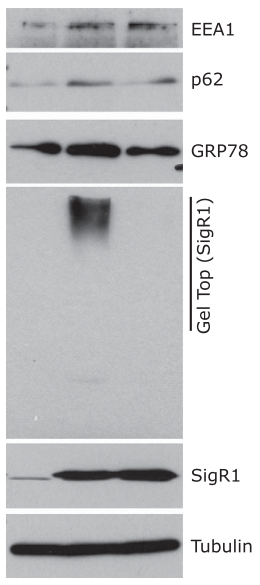

g

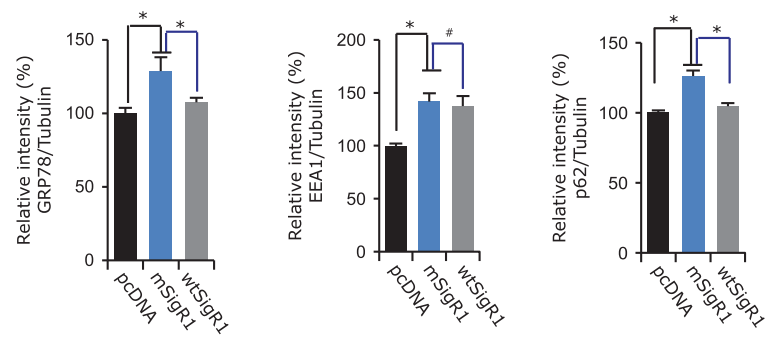

Figure $6 \mathrm{mSigR} 1$ impairs ER to Golgi transport. (a) Cos7 cells showing abnormal SigR1 accumulations (arrows) after mSigR1 transfection and normal ER localization of SigR1 (right) after wtSigR1 transfection. Scale bar, $15 \mu \mathrm{m}$. (b) Cos7 cells were co-transfected with VSVG-GFP together with pcDNA, wtSigR1 or mSigR1. $48 \mathrm{~h}$ after transfection, fluorescence associated with the Golgi complex was photobleached (FRAP, see Materials and Methods) with a high-intensity laser beam. Subsequently, the inward delivery of VSVG-GFP from pre-Golgi intermediates was monitored for the indicated periods of time. Scale bar, $10 \mu \mathrm{m}$. (c) Fluorescence recoveries after photobleaching curves of mSigR1transfected cells show a clear decrease of the mobile fraction as compared to cell transfected with pcDNA or wtSigR1. Error bars indicate the S.E.M.; (right) the comparison of the mobile fractions in pcDNA, wtSigR1 and mSigR1 expressing cells. In the box plots, the line in the middle of the box indicates the median; the top line indicates the 75th quartile, whereas the bottom line indicates the 25th quartile. Whiskers represent the 10th and 90th (upper) percentile, respectively. (d) mSigR1 aggregates (arrowheads) co-localize with the endosomal markers Rab5 (upper) and Rab7 (lower) in Cos-7 cells transfected with wtSigR1 and mSigR1, respectively. Scale bar, $10 \mu \mathrm{m}$. (e) Co-localization of mSigR1 aggregates with the endosomal markers EEA1 and Rab7 in E102Q-SigR1 fALS patients' lymphoblastoid cells compared to healthy controls. Scale bars, $15 \mu$ m. (f) Immunoblot analysis of Cos-7 cells transfected with pcDNA, wtSigR1 and mSigR1. (g) Quantification of band intensities normalized against $\alpha$-tubulin depicted in (f). Values are expressed as mean \pm S.D. from three independent experiments. ${ }^{*} P<0.05$ 
medium (DMEM, Invitrogen), supplemented with 10\% FBS and 1\% antibiotic/antimycotic solution (Invitrogen). Mouse embryonic fibroblasts (MEF) obtained from the GFP-LC3 transgenic mice ${ }^{22}$ were also maintained in DMEM supplemented with $10 \%$ FBS and $1 \%$ antibiotic/anti-mycotic solution. The human epidermoid carcinoma cell line A431 was grown in Dulbecco's modified Eagle's medium (DMEM, Invitrogen) supplemented with 10\% FBS and $0.1 \%$ Gentamycin. NIH-3T3 cells stably expressing GFP-LC3 or tandem mCherry-EGFP-LC3 were cultured in DMEM supplemented with $10 \%$ FBS, $1 \%$ penicillin/streptomycin and puromycin (Sigma Aldrich). Lymphoblasts obtained from E102Q-SigR1 fALS patients and healthy controls were cultured in RPMI 1640 medium containing 20\% FBS, 1\% penicillin/ streptomycin, $1 \%$ L-glutamin and $0.056 \%$ amphotericin. Cells were maintained in a humidified incubator at $37{ }^{\circ} \mathrm{C}$ and $5 \% \mathrm{CO}_{2}$. Generation of $\mathrm{NIH}-3 \mathrm{~T} 3$ cells stably expressing GFP-LC3 or tandem mCherry-EGFP-LC3 with retroviral infection is described elsewhere. ${ }^{14}$

Plasmids and transfection. In order to investigate the molecular mechanisms of E102Q-SigR1-related pathogenesis, cells were transfected to express either wtSigR1 or mSigR1. The vectors for the ectopic expression of wtSigR1 or E102Q-SigR1 have been described already. ${ }^{7}$ The empty pcDNA vector was used as a transfection control. All cell lines were transfected using Lipofectamine 2000 reagent (Invitrogen) according to the manufacturer's recommendations. After $4 \mathrm{~h}$ incubation at $37^{\circ} \mathrm{C}$ and $5 \% \mathrm{CO}_{2}$ the transfection reagent containing medium was replaced by fresh medium and analysis was performed 48h later. VSVG-GFP used for vesicle transport analysis was a kind gift of Prof. Jennifer Lippincott-Schwartz. For proteasome activity analysis, cells were co-transfected with either pcDNA, wtSigR1 or mSigR1.

Post-mortem tissues and Immunohistochemistry. Paraffin sections of human post-mortem lumbar spinal cord $(n=6$ age-matched controls, $n=15$ sALS, $n=9$ C9orf72, $n=4$ FUS; ALS patients) were obtained from the Amsterdam Academic Medical Center (AMC), Division of neuropathology, Department of Pathology ALS Bank (Amsterdam, The Netherlands) Research Code provided by the Medical Ethics Committee of the AMC (Amsterdam, The Netherlands; approved protocol: W11_073). The post-mortem tissues were obtained within 6-12 $\mathrm{h}$ after death. All tissues were used in compliance with the Declaration of Helsinki.

Immunohistochemistry was performed as previously described ${ }^{56}$ on paraffin sections (5-6 $\mu \mathrm{m}$ thickness), de-waxed, rehydrated and heated in Citrate Buffer (pH 6.0), (Thermo Scientific concentrate cat.no.- 005000) for antigen retrieval. Sections were then incubated with primary antibody (Supplementary Table 1) for $1 \mathrm{~h}$ at room temperature. After washing in 0.1M PBS, sections were incubated with appropriate HRP-conjugated secondary antibodies (1:200, Immunologic, Duiven, The Netherlands) for $1 \mathrm{~h}$, followed by 3,3-diaminobenzidine (DAB) visualization (Immunologic). For double immunofluorescence, secondary antibodies conjugated with Alexa fluor 594 and Alexa-fluor 488 were used (Invitrogen), followed by cover slipping with mounting medium containing diamidinophenylindole (DAPI) for nuclear counterstain. Immunoperoxidase stained sections were visualized and photographed using a Zeiss Axioplan microscope with a Zeiss Axiocam HR camera (Zeiss, Oberkochen, Germany) and immunofluorescence was visualized using a Zeiss LSM 700 confocal microscope (Zeiss) and images processed using Zeiss LSM software and Adobe Photoshop CS5.

Immunocytochemistry. HeLa, MCF-7, Cos-7, MEF and NIH-3T3 cells were cultured on $\mu$-dishes (ibidi, GmbH, Planegg/Martinsried, Germany) and transiently transfected either with wtSigR1 or mSigR1. After $48 \mathrm{~h}$ cells were fixed in 4\% PFA and processed for confocal microscopy. Permeabilization with $0.5 \%$ Triton X100 and blocking with $4 \%$ skimmed milk/goat serum was followed by primary antibody incubation overnight at $4{ }^{\circ} \mathrm{C}$. Secondary Alexa488- or Alexa594-conjugated antimouse or anti-rabbit antibodies (Invitrogen) were used for visualization. Nuclei were stained with Hoechst $33342(1 \mu \mathrm{g} / \mathrm{ml})$ or were mounted with DAPI containing fluorescent mounting media (DAKO) and visualized using a Zeiss LSM 700 confocal microscope. Resulting images were processed using the Zeiss LSM software and Adobe Photoshop CS5 (Adobe Systems, San Jose, CA, USA).

Fura-2 calcium imaging. The intracellular calcium ion concentration, $\left[\mathrm{Ca}^{2+}\right]$, was measured using a conventional Fura-2 technique as previously described. ${ }^{11}$ After $48 \mathrm{~h}$ of transfection with pcDNA, wtSigR1 or mSigR1, NSC-34 and MCF-7 cells cultured in glass bottom $\mu$-dishes (ibidi), were loaded with the membranepermeable AM-form of Fura-2 $(1.5 \mathrm{ng} / \mu \mathrm{l}$; Invitrogen) in the presence of pluronic acid
(25\%) for 30 min at $37^{\circ} \mathrm{C}$. Emitted fluorescence at $530 \mathrm{~nm}$ (detected using a PCO (Sensicam: pco.imaging), Kelheim, Germany) in response to alternate excitation at $340 \mathrm{~nm}$ and $380 \mathrm{~nm}$ (using the Polychrome V monochromator; TILL Photonics, Gräfelfing, Germany) was used to measure intracellular $\mathrm{Ca}^{2+}$ concentrations. Data were shown as emission ratios in response to $340 \mathrm{~nm} / 380 \mathrm{~nm}$ excitation. Wholecell calcium measurements of NSC-34 and MCF-7 cells transfected with pcDNA, wtSigR1 or mSigR1 were obtained at room temperature $\left(23-25^{\circ} \mathrm{C}\right)$. During the imaging procedure, cells were kept in a bathing solution containing $100 \mathrm{mM} \mathrm{NaCl}$, $5.4 \mathrm{mM} \mathrm{KCl}, 2 \mathrm{mM} \mathrm{CaCl} 2,1 \mathrm{mM} \mathrm{MgCl} 2,10 \mathrm{mM} \mathrm{HEPES}, 10 \mathrm{MES}, 5.5$ glucose and $\mathrm{pH}$ was adjusted to 7.4 .

ER stress assay. To investigate the ER stress levels in pcDNA, wtSigR1 and mSigR1 expressing cells, we used the ERSE reporter system (Cignal reporter assay kit, Qiagen Cat. No.-CCS-2032L, Hilden, Germany) in combination with the dual luciferase system (Promega Cat.No. - E1910, Madison, WI, USA) according to the manufacturers' protocols. Cells were co-transfected with pcDNA, wtSigR1 and mSigR1 together with the ERSE reporter construct containing the luciferase gene. After $24 \mathrm{~h}$ of incubation, luciferase activity was measured by using the dual luciferase assay for firefly and renilla luciferase detection.

Mitochondrial toxicity assay. Mitochondrial toxicity exerted by mSigR1 was measured using the mitochondrial ToxGlo assay kit (Promega: Cat. No-G8000) according to the manufacturer's protocol. MCF-7 cells were plated on a 96 well plate and transfected with wtSigR1 or mSigR1. $48 \mathrm{~h}$ after transfection, cells were incubated with cytotoxicity reagent at $37^{\circ} \mathrm{C}$ for $30 \mathrm{~min}$ and fluorescence was measured at $520 \mathrm{~nm}$. This value represents the membrane integrity (MI). Thereafter the same plate was equilibrated at room temperature and then incubated with the ATP detection substrate; luminescence was measured after $5 \mathrm{~min}-1 \mathrm{~h}$ for the detection of ATP production.

Transmission electron microscopy (TEM). MCF-7 and NSC-34 cells were transfected with pcDNA, wtSigR1 or mSigR1 as described above. Cells were collected by scraping, suspended lymphoblast cultures from healthy controls and ALS patients were collected by brief centrifugation ( 5000 r.p.m. for $5 \mathrm{~min}$ ) and then washed in $0.1 \mathrm{M}$ phosphate buffer and immediately fixed with $2.5 \%$ glutaraldehyde in $0.1 \mathrm{M}$ phosphate buffer for $24 \mathrm{~h}$ followed by washing in buffer for another $24 \mathrm{~h}$. Cell pellets were collected by centrifugation (1000 r.p.m., $5 \mathrm{~min}$ ) and embedded in $2 \%$ agarose (at $60^{\circ} \mathrm{C}$; Fluka \#05073). Small blocks of embedded cells were sliced and post-fixed in $2.5 \%$ glutaraldehyde for $24 \mathrm{~h}$ followed by washing in $0.1 \mathrm{M}$ phosphate buffer for $24 \mathrm{~h}$. Agarose blocks were then incubated in 1\% OsO4 (in $0.2 \mathrm{M}$ phosphate buffer) for $3 \mathrm{~h}$, washed twice in distilled water and dehydrated with using ascending alcohol concentrations (i.e., 25, 35, 50, 70, 85, 95 and 100\%; each step for $5 \mathrm{~min}$ ). Dehydrated blocks were incubated in propylenoxide followed by subsequent 20 min incubation in a 1:1 mixture of epon (47.5\% glycidether, $26.5 \%$ dodenylsuccinic acid anhydride, $24.5 \%$ methylnadic anhydride and $1.5 \%$ Tris (dimethylaminomethyl) phenol) and propylenoxide. The samples were then incubated in epoxy resin for $1 \mathrm{~h}$ at room temperature followed by polymerization $\left(28^{\circ} \mathrm{C}\right.$ for $8 \mathrm{~h}, 80^{\circ} \mathrm{C}$ for $2.5 \mathrm{~h}$ and finally at room temperature for $4 \mathrm{~h}$ ). Ultra-thin sections $(70 \mathrm{~nm})$ were mounted on grids for electron microscopy and examined using a Philips CM10 transmission electron microscope (Philips, Amsterdam, The Netherlands) as already described. ${ }^{11}$

Fluorescence recovery after photobleaching (FRAP). For live cell imaging experiments, Cos7 cells were seeded on glass-bottomed dishes $(\varnothing 6 \mathrm{~cm})$ and co-transfected either with pcDNA/VSVGts045-GFP, wtSigR1/VSVGts045-GFP or mSigR1/ VSVGts045-GFP. VSVGts045-GFP was allowed to accumulate in the ER at $42{ }^{\circ} \mathrm{C}$ for $8 \mathrm{~h}$ and cells were imaged on an Axio Observer $\mathrm{Z1}$ inverted microscope equipped with heating stage and $\mathrm{CO}_{2}$ controller (Zeiss) maintained at a constant temperature of $32{ }^{\circ} \mathrm{C}$. A portion of the ER $(\varnothing \sim 3.84 \mu \mathrm{m})$ in the periphery of the cell was photobleached using a $405 \mathrm{~nm}$ laser driven by the UGA-40 control unit (Rapp Opto Electronic GmbH, Wedel, Germany). The recovery of the fluorescent signal was monitored by imaging the cells every second for $15 \mathrm{~min}$. Imaging was done using an Evolve EM-CCD camera driven by ZEN software (Zeiss). For all experiments the bleached area and the duration of the laser impulse were kept constant. The extent of recovery of the fluorescent signal was determined using ImageJ to measure the average pixel intensity values within three distinct regions of interest: ROl1: bleached area, ROI2: unbleached area within the cell and ROI3: background. Normalized FRAP recovery curves and the mobile fraction were calculated using the program easy FRAP. 
Live cell imaging to analyze RFP-GFP-LC3 fusion. To analyze the dynamics of the RFP-GFP-LC3 fusion protein, GFP and RFP channels were acquired every minute for up to $4 \mathrm{~h}$ using the imaging system described above. The extent of autophagosome maturation was determined by measuring the co-localization of the GFP and RFP signals as expressed by the Pearson's coefficient using ZEN software.
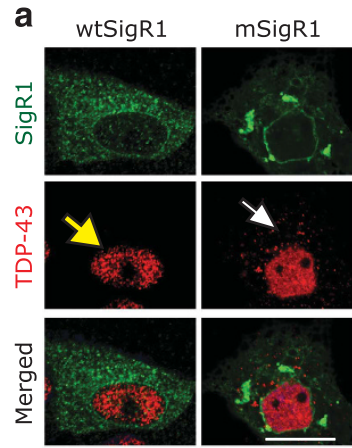
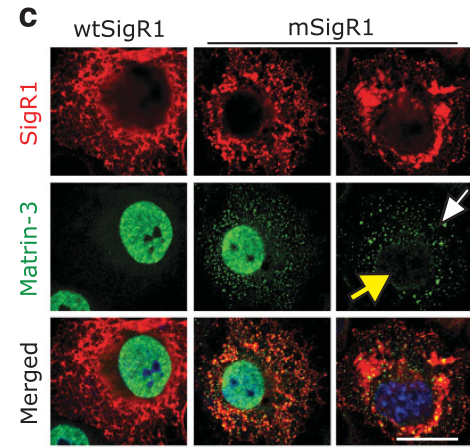
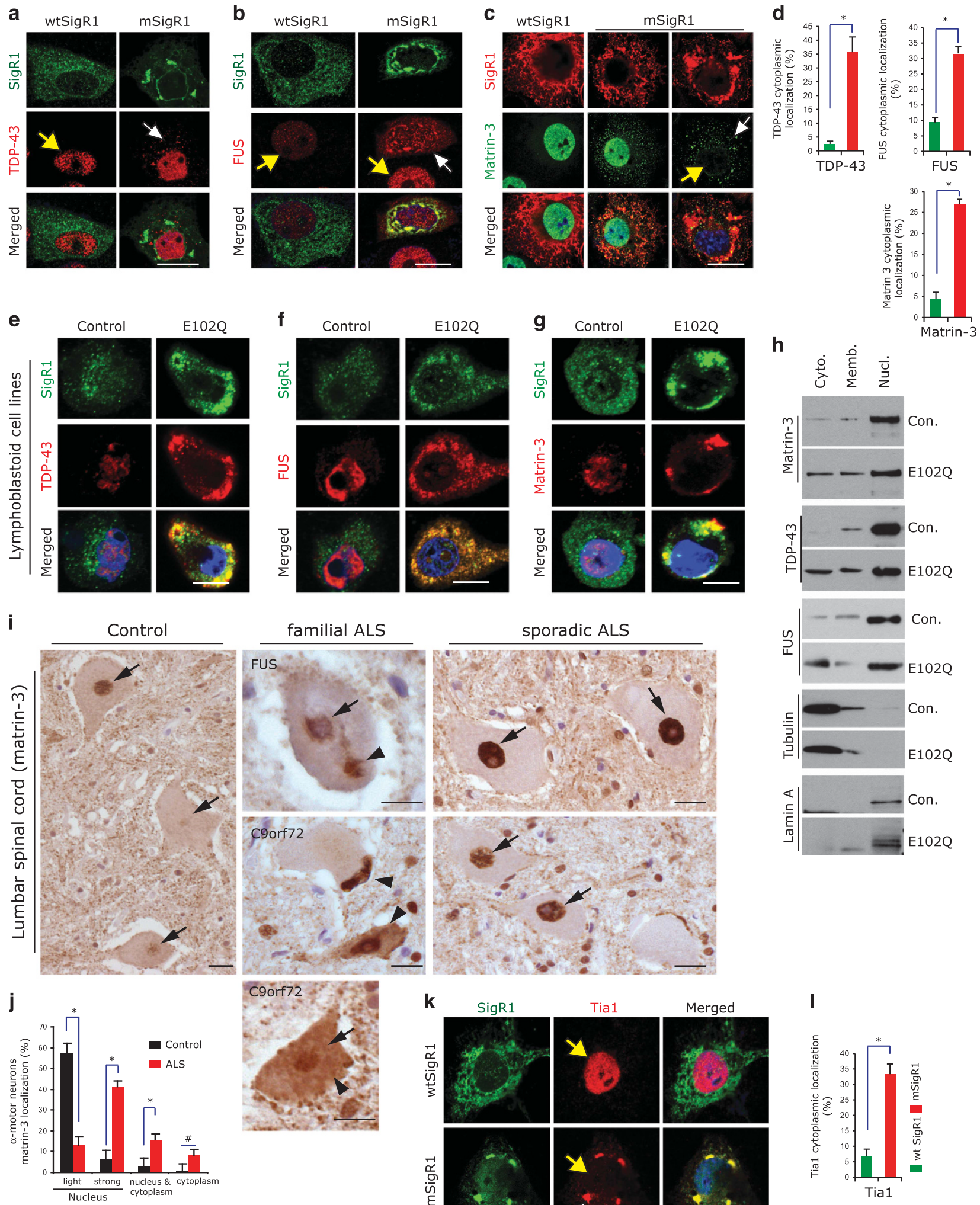

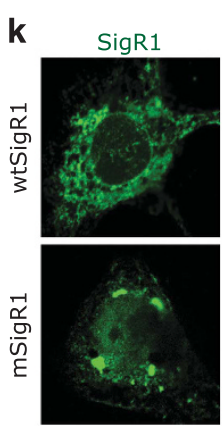

h
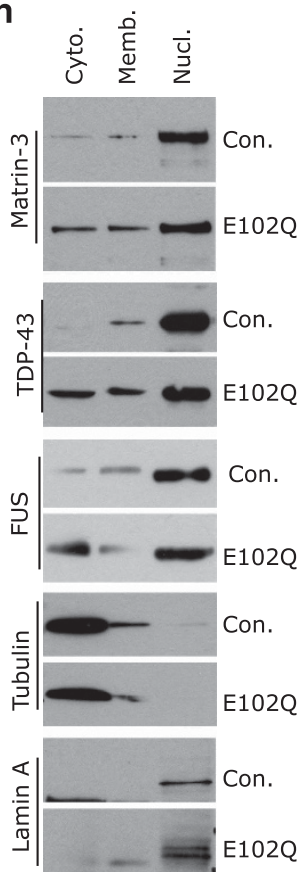

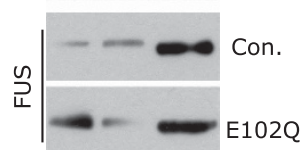


Immunoblot analysis. Cells were washed twice with ice-cold PBS and scraped off the culture plate. After centrifugation at 6000 r.p.m. for $5 \mathrm{~min}$ and removal of the supernatant, cell pellets were re-suspended in lysis buffer (0.5\% Triton X-100 in PBS, $0.5 \mathrm{mM}$ PMSF and complete protease inhibitor mixture, Roche Applied Sciences, Mannheim, Germany) and incubated on ice for $30 \mathrm{~min}$ followed by a sonification with an amplitude of $8 \%$ for $10 \mathrm{~s}$. Clear lysates were obtained after centrifugation for $5 \mathrm{~min}$ at 6000 r.p.m. Protein concentrations were determined using the BCA method (Molecular Probes). Equal amounts of protein were boiled for $5 \mathrm{~min}$ in $2 \times$ SDS sample buffer and subjected to 10 or $12 \%$ SDSPAGE electrophoresis at $80 \mathrm{~mA}$ before transferred to a polyvinylidenedifluoride membrane, which had to be activated in methanol before. Transfer lasts $1 \mathrm{~h}$ and $30 \mathrm{~min}$ at $350 \mathrm{~mA}$ and was followed by blocking in $4 \%$ skimmed milk in $0.05 \%$ Tween 20/Tris-buffered saline (TBS-T) for $30 \mathrm{~min}$ before incubation with primary antibody. The dilutions for primary antibodies are described in Supplementary Table 1. After incubating the primary antibody overnight at $4{ }^{\circ} \mathrm{C}$ under gently shaking, membranes were washed three times with TBS-T for 10 min each and incubated with the appropriate horseradish peroxidase-conjugated secondary antibody for $1 \mathrm{~h}$ (antibody dilution 1:10 000) followed by the same washing procedure. Mutant SigR1 aggregates were detected by using dot blot analyis. For this, lymphoblastoid cell lysates were directly brought on a nitrocellulose membrane and after applying a vacuum, membranes were processed following our established western blot protocol. Immunoreactive proteins were detected by enhanced chemiluminescence (Amersham Biosciences, Mannheim, Germany). Densitometric quantification of the band intensity was normalized to tubulin levels using Adobe Photoshop CS5.

EGFR endocytosis, starvation and degradation assay. For EGFR endocytosis and degradation analysis, A431 cells were starved in DMEM without

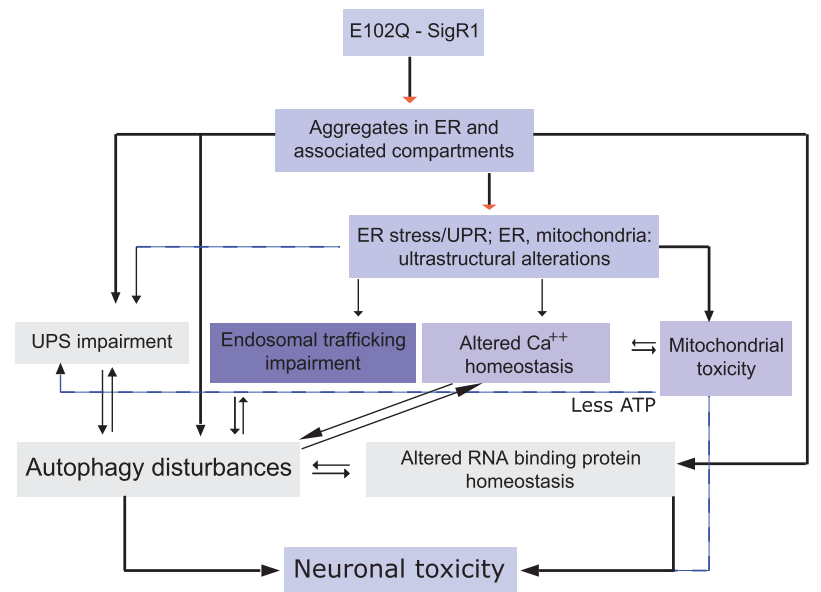

Figure 8 Illustration showing the proposed mechanisms of ALS pathogenesis associated with the $\mathrm{E} 102 \mathrm{Q}$ mutation in SigR1 serum for $4 \mathrm{~h}$. After starvation, cells were treated with EGF $(100 \mathrm{mg})$ to stimulate EGFR endocytosis. Cells were then collected at various time points (as indicated in Figures $3 \mathrm{l}$ and $\mathrm{m}$ ) and lysed in RIPA buffer $(50 \mathrm{mM}$ Tris-HClpH 7.5, $1 \%$ Triton X-100, $150 \mathrm{mM} \mathrm{NaCl}, 1 \mathrm{mM}$ EDTA and $0.1 \%$ Na deoxycholate) containing protease inhibitor. Protein extracts were resolved by SDS-PAGE and immunoblotted using anti-EGFR antibody.

EGFR surface biotinylation assay. Surface biotinylation assays were performed as previously described ${ }^{14}$ with minor modifications. Briefly, transfected A431 cells were starved in DMEM without serum for $4 \mathrm{~h}$. After starvation, cells were treated with EGF to stimulate EGFR endocytosis and then preceded for surface biotinylation assay according to the manufacturer's protocol.

Mito-red staining. MCF-7 cells were grown on coverslips and transfected with wtSigR1 or mSigR1. After $48 \mathrm{~h}$ cells were incubated with the mito-red dye for $10 \mathrm{~min}$ at $37^{\circ} \mathrm{C}$ and processed according to the manufacturer's protocol for adherent cells. For quantification of enlarged mitochondria mito-red stained cells were visualized with the LSM 700 confocal microscope using the $40 x$ lens. Enlarged mitochondria (showing accumulation of mito-red staining and larger in size than normal mitochondria) from 10 random fields and at least capturing 8-10 cells per field of view were counted manually.

CYTO-ID Autophagy detection. MCF-7 cells were grown on coverslips and transfected with wtSigR1 or mSigR1. After $48 \mathrm{~h}$ cells were incubated with the CYTO-ID dye (Enzo, Lausen, Switzerland) for $30 \mathrm{~min}$ at $37^{\circ} \mathrm{C}$ and processed according to the manufacturer's protocol for adherent cells. Lymphoblastoid cells derived from E102Q-SigR1 fALS patients and healthy controls were processed following the manufacturer's instructions for suspension cells. Both were analyzed by confocal microscopy.

Enzyme activity assays and cell viability. NSC-34 cells were transfected with pcDNA, wtSigR1 or mSigR1 as described previously. After $48 \mathrm{~h}$ incubation, cells were washed twice with ice-cold PBS and scraped off the culture plate using a cell scraper. Cell pellets were lysed in homogenization buffer for 15 min (10 mM Tris-HCl (ph 7.4), 1 mM EDTA, 5 mM DTT, 5 mM ATP, 20\% glycerol and complete protease inhibitor mixture, Roche Applied Sciences) and processed for the proteasome activity assay (Jana). Proteasome activity was measured by using the proteasome substrate Suc-Leu-Leu-Val-Tyr-AMC. Lymphoblasts obtained from patients or healthy control individuals were processed for the caspase-3 activity assay following the same protocol using the caspase-3 substrate N-AcetylAsp-Glu-Val-Asp-7 amido-4-methylcoumarin.

Cell viability (mitochondrial activity) was determined by using Alamar Blue. Therefore, NSC34 cells were grown in 24 well plates and transfected according to the protocol described before. Alamar Blue was added to the medium in a 1:10 dilution and absorbance was measured after $2 \mathrm{~h}$ at $570 \mathrm{~nm}$.

Cellular fractionation. The subcellular distributions of distinct proteins were determined by using a subcellular fractionation kit following the manufacturer's protocol (Thermo Scientific/Life Technologies) and analyzed by immunoblotting. The

Figure $7 \mathrm{mSigR} 1$ accumulation leads to altered RNA-binding protein homeostasis. (a) Co-staining of SigR1 and TDP-43 in MCF-7 cells transfected with either wtSigR1 or mSigR1. Note the minor cytoplasmic TDP-43 accumulations (white arrow) without co-localization with SigR1, in contrast to the more pronounced TDP-43 aggregates colocalizing with SigR1 in E102Q-SigR1 fALS lymphoblastoid cell depicted in e. Scale bar, $15 \mu \mathrm{m}$. (b) Translocation of FUS from the nucleus (yellow arrow) to the cytoplasm (white arrow) and co-localization with SigR1 aggregates in MCF-7 cells expressing mSigR1 compared to wtSigR1-transfected cells. Scale bar, $10 \mu \mathrm{m}$. (c) Immunofluorescence staining of SigR1 and matrin-3 in MCF-7 cells expressing wtSigR1 or mSigR1. Note the cytoplasmic accumulations (white arrow) along with the loss of nuclear matrin-3 (yellow arrow) corresponding to the higher amount of globular mSigR1 aggregates. Scale bar, $10 \mu \mathrm{m}$. (d) Quantification ( $n=3$ each) of the experiments illustrated in (a-c). ${ }^{*} P<0.05$. (e-g) Nuclear loss of TDP43 (e), FUS (f) and matrin-3 (g) and their co-localization with mSigR1 aggregates in E102Q-SigR1 fALS lymphoblastoid cells compared to healthy controls. Scale bar, $10 \mu \mathrm{m}$. (h) Immunoblot analysis of subcellular fractions obtained from E102Q-SigR1 patients' lymphoblastoid cell lines compared to healthy controls ( $n=2$ for fALS; $n=3$ for control). Matrin-3, FUS and TDP-43 distributions are shown in the cytoplasmic (Cyto.), membrane (Memb.) and nuclear (Nucl.) fractions. Note the translocation of matrin3 from the nucleus to the cytoplasm in E102Q-SigR1 patients' lymphoblastoid cells. Tubulin is used as a loading control and Lamin A as a positive control for the nuclear fraction. (i) Immunohistochemical analysis of lumbar $\alpha$-MNs using matrin-3 antibody in SALS and fALS compared to normal controls. Cytoplasmic accumulation (arrowheads, middle) of matrin-3 in $\alpha$-MNs of fALS patients harbouring FUS and C9ORF72 mutations. Strong nuclear immunoreactivity of matrin-3 (arrows) is evident in sALS $\alpha$-MNs (right), whereas the control shows an overall weaker nuclear matrin-3 signal (arrows, left). Paraffin sections, DAB-immunohistochemistry; scale bars, $20 \mu \mathrm{m}$. (j) Quantification of immunohistochemical data illustrated in I; for the immunohistochemical analysis $n=12 \mathrm{sALS} ; n=8$ (C9ORF72); $n=4$ (FUS) and $n=4$ control cases were examined. ${ }^{*} P<0.05$, while \# denotes absence of significance (k) loss of nuclear Tia1 (yellow arrows) and accumulation of cytoplasmic Tia-1 (white arrow in the middle panel) positive stress granules co-localized with mSigR1 aggregates in MCF-7 cells. Scale bar, $15 \mu \mathrm{m}$. (I) Quantification of data shown in (k). ${ }^{*} P<0.05$ 
subcellular fractionation was performed with patients' and control lymphoblasts as well as with NSC34 cells transfected according to the described procedure.

Statistical analysis. We used the unpaired Student's t-test for comparison between two sample groups. Values were expressed as mean \pm S.D. from three independent experiments. For $\mathrm{Ca}^{2+}$ measurements results were expressed as means \pm S.E.M. of $\sim 30$ cells. For the FRAP and co-localization experiments, we used the Mann-Whitney U-test. For both statistical tests, differences between the compared experimental conditions were regarded as significant when ${ }^{*} P<0.05$, ${ }^{* *} P<0.005$.

\section{Conflict of Interest}

The authors declare no conflict of interest.

Acknowledgements. We thank A Knischewski, H Wiederholt, E Pascual, C Krude and H Mader (Institute of Neuropathology, RWTH Aachen University) for their technical support and I Kurth and I Diepolder (Institute of Human Genetics, RWTH Aachen University) for the generation and maintenance of PLCs. We also thank Dr. Amr M Al-Saif (Laboratory of Neurogenetics, Neuroscience Research Center, $\mathrm{NIH}$; USA) and V Kumar (Max Planck Institute for Biological Cybernetics, Tuebingen, Germany) for their valuable comments and image analysis and S Gruender (Institute of Physiology, RWTH Aachen University) and his laboratory members for confocal microscopy and other generous help. We sincerely thank Stichting ALS Nederland and ALS Centre Netherlands (AE) for our ALS research support. Finally, we thank B Luescher (Institute of Biochemistry and Molecular Biology, RWTH Aachen University) and his laboratory members for generous help. This work was supported by grants of the Interdisciplinary Centre for Clinical Research (IZKF Aachen) (N1-1, N5-3), by the German Motor Neuron Disease Network (MND-Net; 360644) and by the German Charcot-Marie-Tooth Disease (CMT-Net; 01GM1511D) funded by the German ministry of education and research (BMBF, Bonn, Germany) to JW and of the German Myopathy Society (DGM) and the Initiative Therapieforschung ALS e.V. to AG and JW.

\section{Author contributions}

$A G$ raised the hypotheses and designed the experiments. Most experimental work was performed by AD, TV and AY. Live cell imaging and FRAP analysis were done by AS. RT-PCR was performed by SJ. Electron microscopy was performed by IK and AR. Calcium imaging was performed by AG, DW and YT. MEF cells from the GFP-LC3 transgenic mouse and $\mathrm{NIH}-3 \mathrm{~T} 3$ cell lines stably expressing GFP-LC3 and tandemly fused mCherry-GFP-LC3, respectively, were generated by JV and MD. Confocal imaging was performed by AG. The schematic representation SigR1 model was designed by AS. ALS cases were provided by EA, DT. The ALS tissues were prepared by JA. The SigR1 E102Q fALS cases were provided by SB. The manuscript was written by $A G, A D$ and JW. $A G$ and JW supervised the entire project. All authors discussed results and commented on the manuscript.

1. Renton AE, Chio A, Traynor BJ. State of play in amyotrophic lateral sclerosis genetics. Nat Neurosci 2014; 17: 17-23.

2. Ling SC, Polymenidou M, Cleveland DW. Converging mechanisms in ALS and FTD: disrupted RNA and protein homeostasis. Neuron 2013; 79: 416-438.

3. Polymenidou M, Lagier-Tourenne C, Hutt KR, Bennett CF, Cleveland DW, Yeo GW. Misregulated RNA processing in amyotrophic lateral sclerosis. Brain Res 2012; 1462: 3-15.

4. Urushitani M, Sato T, Bamba H, Hisa Y, Tooyama I. Synergistic effect between proteasome and autophagosome in the clearance of polyubiquitinated TDP-43. J Neurosci Res 2010; 88 : 784-797.

5. Wolozin B. Regulated protein aggregation: stress granules and neurodegeneration. $\mathrm{Mol}$ Neurodegener 2012; 7: 56

6. Hayashi T, Su TP. Sigma-1 receptor chaperones at the ER-mitochondrion interface regulate $\mathrm{Ca}(2+)$ signaling and cell survival. Cell 2007; 131: 596-610.

7. Al-Saif A, Al-Mohanna F, Bohlega S. A mutation in sigma-1 receptor causes juvenile amyotrophic lateral sclerosis. Ann Neurol 2011; 70: 913-919.

8. Luty AA, Kwok JB, Dobson-Stone C, Loy CT, Coupland KG, Karlstrom H et al. Sigma nonopioid intracellular receptor 1 mutations cause frontotemporal lobar degeneration-motor neuron disease. Ann Neurol 2010; 68: 639-649.

9. Gregianin E, Pallafacchina G, Zanin S, Crippa V, Rusmini P, Poletti A et al. Loss-of-function mutations in the SIGMAR1 gene cause distal hereditary motor neuropathy by impairing ER-mitochondria tethering and Ca2+ signaling. Hum Mol Genet 2016; 25: 3741-3753.
10. Nguyen L, Lucke-Wold BP, Mookerjee SA, Cavendish JZ, Robson MJ, Scandinaro AL et al. Role of sigma-1 receptors in neurodegenerative diseases. J Pharmacol Sci 2015; 127: 17-29.

11. Prause J, Goswami A, Katona I, Roos A, Schnizler M, Bushuven E et al. Altered localization, abnormal modification and loss of function of Sigma receptor-1 in amyotrophic lateral sclerosis. Hum Mol Genet 2013; 22: 1581-1600.

12. Mavlyutov TA, Epstein ML, Verbny YI, Huerta MS, Zaitoun I, Ziskind-Conhaim L et al. Lack of sigma-1 receptor exacerbates ALS progression in mice. Neuroscience 2013; 240: 129-134.

13. Bernard-Marissal N, Medard JJ, Azzedine H, Chrast R. Dysfunction in endoplasmic reticulum-mitochondria crosstalk underlies SIGMAR1 loss of function mediated motor neuron degeneration. Brain 2015; 138: 875-890.

14. Vollrath JT, Sechi A, Dreser A, Katona I, Wiemuth D, Vervoorts J et al. Loss of function of the ALS protein SigR1 leads to ER pathology associated with defective autophagy and lipid raft disturbances. Cell Death Dis 2014; 5: e1290.

15. Mancuso R, Olivan S, Rando A, Casas C, Osta R, Navarro X. Sigma-1R agonist improves motor function and motoneuron survival in ALS mice. Neurotherapeutics 2012; 9: 814-826.

16. Schmidt HR, Zheng S, Gurpinar E, Koehl A, Manglik A, Kruse AC. Crystal structure of the human sigma1 receptor. Nature 2016; 532: 527-530.

17. Tagashira H, Shinoda Y, Shioda N, Fukunaga K. Methyl pyruvate rescues mitochondrial damage caused by SIGMAR1 mutation related to amyotrophic lateral sclerosis. Biochim Biophys Acta 2014; 1840: 3320-3334.

18. Pabba M, Wong AY, Ahlskog N, Hristova E, Biscaro D, Nassrallah W et al. NMDA receptors are upregulated and trafficked to the plasma membrane after sigma-1 receptor activation in the rat hippocampus. J Neurosci 2014; 34: 11325-11338.

19. Hamasaki M, Furuta N, Matsuda A, Nezu A, Yamamoto A, Fujita N et al. Autophagosomes form at ER-mitochondria contact sites. Nature 2013; 495: 389-393.

20. Klionsky DJ, Cuervo AM, Seglen PO. Methods for monitoring autophagy from yeast to human. Autophagy 2007; 3: 181-206.

21. Rubinsztein DC, Cuervo AM, Ravikumar B, Sarkar S, Korolchuk V, Kaushik S et al. In search of an "autophagomometer". Autophagy 2009; 5: 585-589.

22. Mizushima N, Yamamoto A, Matsui M, Yoshimori T, Ohsumi Y. In vivo analysis of autophagy in response to nutrient starvation using transgenic mice expressing a fluorescent autophagosome marker. Mol Biol cell 2004; 15: 1101-1111.

23. Kimura S, Fujita N, Noda T, Yoshimori T. Monitoring autophagy in mammalian cultured cells through the dynamics of LC3. Methods Enzymol 2009; 452: 1-12.

24. Nehls S, Snapp EL, Cole NB, Zaal KJ, Kenworthy AK, Roberts TH et al. Dynamics and retention of misfolded proteins in native ER membranes. Nat Cell biol 2000; 2: 288-295.

25. Presley JF, Cole NB, Schroer TA, Hirschberg K, Zaal KJ, Lippincott-Schwartz J. ER-to-Golgi transport visualized in living cells. Nature 1997; 389: 81-85.

26. Fasana E, Fossati M, Ruggiano A, Brambillasca S, Hoogenraad CC, Navone F et al. A VAPB mutant linked to amyotrophic lateral sclerosis generates a novel form of organized smooth endoplasmic reticulum. FASEB J 2010; 24: 1419-1430.

27. Wong AY, Hristova E, Ahlskog N, Tasse LA, Ngsee J, Chudalayandi $P$ et al. Aberrant subcellular dynamics of sigma-1 receptor mutants underlying neuromuscular diseases. $\mathrm{Mol}$ Pharmacol 2016; 90: 238-253.

28. Farrawell NE, Lambert-Smith IA, Warraich ST, Blair IP, Saunders DN, Hatters DM et al. Distinct partitioning of ALS associated TDP-43, FUS and SOD1 mutants into cellular inclusions. Sci Rep 2015; 5: 13416.

29. Walsh MJ, Cooper-Knock J, Dodd JE, Stopford MJ, Mihaylov SR, Kirby J et al. Invited review: decoding the pathophysiological mechanisms that underlie RNA dysregulation in neurodegenerative disorders: a review of the current state of the art. Neuropathol Appl Neurobiol 2015; 41: 109-134.

30. Johnson JO, Pioro EP, Boehringer A, Chia R, Feit H, Renton AE et al. Mutations in the Matrin 3 gene cause familial amyotrophic lateral sclerosis. Nat Neurosci 2014; 17: 664-666.

31. Senderek J, Garvey SM, Krieger M, Guergueltcheva V, Urtizberea A, Roos A et al, Autosomal-dominant distal myopathy associated with a recurrent missense mutation in the gene encoding the nuclear matrix protein, matrin 3. Am J Hum Genet 2009; 84: 511-518.

32. Muller TJ, Kraya T, Stoltenburg-Didinger G, Hanisch F, Kornhuber M, Stoevesandt D et al. Phenotype of matrin-3-related distal myopathy in 16 German patients. Ann Neurol 2014; 76 : 669-680.

33. Salton M, Elkon R, Borodina T, Davydov A, Yaspo ML, Halperin E et al. Matrin 3 binds and stabilizes mRNA. PloS One 2011; 6: e23882.

34. Dewey CM, Cenik B, Sephton CF, Johnson BA, Herz J, Yu G. TDP-43 aggregation in neurodegeneration: are stress granules the key? Brain Res 2012; 1462: 16-25.

35. Nishimura AL, Mitne-Neto M, Silva HC, Richieri-Costa A, Middleton S, Cascio D et al. A mutation in the vesicle-trafficking protein VAPB causes late-onset spinal muscular atrophy and amyotrophic lateral sclerosis. Am J Hum Genet 2004; 75: 822-831.

36. Funke AD, Esser M, Kruttgen A, Weis J, Mitne-Neto M, Lazar M et al. The p.P56S mutation in the VAPB gene is not due to a single founder: the first European case. Clin Genet 2010; 77: 302-303.

37. Kuijpers $M$, van Dis V, Haasdijk ED, Harterink M, Vocking K, Post JA et al. Amyotrophic lateral sclerosis (ALS)-associated VAPB-P56S inclusions represent an ER quality control compartment. Acta Neuropathol Commun 2013; 1: 24.

38. Senderek J, Krieger M, Stendel C, Bergmann C, Moser M, Breitbach-Faller N et al. Mutations in SIL1 cause Marinesco-Sjogren syndrome, a cerebellar ataxia with cataract and myopathy. Nat Genet 2005; 37: 1312-1314. 
39. Evgrafov OV, Mersiyanova I, Irobi J, Van Den Bosch L, Dierick I, Leung CL et al. Mutant small heat-shock protein 27 causes axonal Charcot-Marie-Tooth disease and distal hereditary motor neuropathy. Nat Genet 2004; 36: 602-606.

40. Krieger M, Roos A, Stendel C, Claeys KG, Sonmez FM, Baudis M et al. SIL1 mutations and clinical spectrum in patients with Marinesco-Sjogren syndrome. Brain 2013; 136: 3634-3644.

41. Filezac de L'Etang A, Maharjan N, Cordeiro Brana M, Ruegsegger C, Rehmann R, Goswami A et al. Marinesco-Sjogren syndrome protein SIL1 regulates motor neuron subtype-selective ER stress in ALS. Nat Neurosci 2015; 18: 227-238.

42. Watanabe S, llieva H, Tamada H, Nomura H, Komine O, Endo $F$ et al. Mitochondriaassociated membrane collapse is a common pathomechanism in SIGMAR1- and SOD1-linked ALS. EMBO Mol Med 2016, 8: 1421-1437.

43. Omi T, Tanimukai H, Kanayama D, Sakagami $\mathrm{Y}$, Tagami S, Okochi M et al. Fluvoxamine alleviates ER stress via induction of Sigma-1 receptor. Cell Death Dis 2014; 5: e1332.

44. Suzuki H, Kanekura K, Levine TP, Kohno K, Olkkonen VM, Aiso S et al. ALS-linked P56S-VAPB, an aggregated loss-of-function mutant of VAPB, predisposes motor neurons to ER stress-related death by inducing aggregation of co-expressed wild-type VAPB. J Neurochem 2009; 108: 973-985.

45. Suzuki $H$, Matsuoka M. Amyotrophic lateral sclerosis-linked mutant VAPB enhances TDP-43-induced motor neuronal toxicity. J Neurochem 2011; 119: 1099-1107.

46. Halliday G, Bigio EH, Cairns NJ, Neumann M, Mackenzie IR, Mann DM. Mechanisms of disease in frontotemporal lobar degeneration: gain of function versus loss of function effects. Acta Neuropathol 2012; 124: 373-382.

47. Roos A, Buchkremer S, Kollipara L, Labisch T, Gatz C, Zitzelsberger M et al. Myopathy in Marinesco-Sjogren syndrome links endoplasmic reticulum chaperone dysfunction to nuclear envelope pathology. Acta neuropathol 2014; 127: 761-777.

48. Tsai SY, Chuang JY, Tsai MS, Wang XF, Xi ZX, Hung JJ et al. Sigma-1 receptor mediates cocaine-induced transcriptional regulation by recruiting chromatin-remodeling factors at the nuclear envelope. Proc Natl Acad Sci USA 2015; 112: E6562-E6570.

49. Tran D, Chalhoub A, Schooley A, Zhang W, Ngsee JK. A mutation in VAPB that causes amyotrophic lateral sclerosis also causes a nuclear envelope defect. J Cell Sci 2012; 125: 2831-2836.
50. Bezprozvanny I. Calcium signaling and neurodegenerative diseases. Trends Mol Med 2009; 15: $89-100$.

51. Krols M, van Isterdael G, Asselbergh B, Kremer A, Lippens S, Timmerman V et al. Mitochondria-associated membranes as hubs for neurodegeneration. Acta Neuropathol 2016; 131: 505-523.

52. Wang X, Fan H, Ying Z, Li B, Wang H, Wang G. Degradation of TDP-43 and its pathogenic form by autophagy and the ubiquitin-proteasome system. Neurosci Lett 2010; 469: 112-116.

53. Ryu HH, Jun MH, Min KJ, Jang DJ, Lee YS, Kim HK et al. Autophagy regulates amyotrophic lateral sclerosis-linked fused in sarcoma-positive stress granules in neurons. Neurobiol Aging 2014; 35: 2822-2831.

54. Moloney C, Rayaprolu S, Howard J, Fromholt S, Brown H, Collins M et al. Transgenic mice overexpressing the ALS-linked protein Matrin 3 develop a profound muscle phenotype. Acta Neuropathol Commun 2016; 4: 122.

55. Saccon RA, Bunton-Stasyshyn RK, Fisher EM, Fratta P. Is SOD1 loss of function involved in amyotrophic lateral sclerosis? Brain 2013; 136: 2342-2358.

56. Jesse CM, Bushuven E, Tripathi P, Chandrasekar A, Simon CM, Drepper C et al. ALS-associated endoplasmic reticulum proteins in denervated skeletal muscle: implications for motor neuron disease pathology. Brain Pathol 2016 (doi:10.1111/bpa.12453; e-pub ahead of print).

(c) (i) This work is licensed under a Creative Commons Attribution 4.0 International License. The images or other third party material in this article are included in the article's Creative Commons license, unless indicated otherwise in the credit line; if the material is not included under the Creative Commons license, users will need to obtain permission from the license holder to reproduce the material. To view a copy of this license, visit http:// creativecommons.org/licenses/by/4.0/

(C) The Author(s) 2017

Supplementary Information accompanies this paper on Cell Death and Differentiation website (http://www.nature.com/cdd) 